\title{
The structure of the Cepheus E protostellar outflow: The jet, the bowshock, and the cavity ${ }^{\star}$
}

\author{
B. Lefloch ${ }^{1,2}$, A. Gusdorf ${ }^{3,4}$, C. Codella ${ }^{5}$, J. Eislöffel ${ }^{6}$, R. Neri $^{7}$, A. I. Gómez-Ruiz ${ }^{8}$, R. Güsten ${ }^{9}$, \\ S. Leurini ${ }^{9}$, C. Risacher ${ }^{9}$, and M. Benedettini ${ }^{10}$
}

1 Univ. Grenoble Alpes, IPAG, 38000 Grenoble, France e-mail: Bertrand.Lefloch@obs.ujf-grenoble.fr

2 CNRS, IPAG, 38000 Grenoble, France

3 LERMA, Observatoire de Paris, PSL Research University, CNRS, UMR 8112, 75014 Paris, France

${ }^{4}$ Sorbonne Universités, UPMC Univ. Paris 6, UMR 8112, LERMA, 75005 Paris, France

5 INAF, Osservatorio Astrofisico di Arcetri, Largo Enrico Fermi 5, 50125 Firenze, Italy

6 Thüringer Landessternwarte, Tautenburg, Sternwarte 5, 07778 Tautenburg, Germany

7 Institut de Radioastronomie Millimétrique, Domaine Universitaire, 38406 St.-Martin-d'Hères, France

${ }^{8}$ Instituto Nacional de Astrofísica, Optica y Electrónica, Luis E. Erro 1, Tonantzintla, CP 72840 Puebla, Mexico

9 Max Planck Institut für Radioastronomie, Auf dem Hügel 69, 53121 Bonn, Germany

10 INAF - Istituto di Astrofisica e Planetologia Spaziali, via del Fosso del Cavaliere 100, 00133 Roma, Italy

Received 15 December 2014 / Accepted 19 June 2015

\section{ABSTRACT}

Context. Protostellar outflows are a crucial ingredient of the star-formation process. However, the physical conditions in the warm outflowing gas are still poorly known.

Aims. We present a multi-transition, high spectral resolution CO study of the outflow of the intermediate-mass Class 0 protostar Cep E-mm. The goal is to determine the structure of the outflow and to constrain the physical conditions of the various components in order to understand the origin of the mass-loss phenomenon.

Methods. We have observed the $J=12-11, J=13-12$, and $J=16-15$ CO lines at high spectral resolution with SOFIA/GREAT and the $J=5-4, J=9-8$, and $J=14-13$ CO lines with HIFI/Herschel towards the position of the terminal bowshock HH377 in the southern outflow lobe. These observations were complemented with maps of CO transitions obtained with the IRAM $30 \mathrm{~m}$ telescope $(J=1-0,2-1)$, the Plateau de Bure interferometer $(J=2-1)$, and the James Clerk Maxwell Telescope $(J=3-2,4-3)$.

Results. We identify three main components in the protostellar outflow: the jet, the cavity, and the bowshock, with a typical size of $1.7^{\prime \prime} \times 21^{\prime \prime}, 4.5^{\prime \prime}$, and $22^{\prime \prime} \times 10^{\prime \prime}$, respectively. In the jet, the emission from the low- $J$ CO lines is dominated by a gas layer at $T_{\text {kin }}=$ $80-100 \mathrm{~K}$, column density $N(\mathrm{CO})=9 \times 10^{16} \mathrm{~cm}^{-2}$, and density $n\left(\mathrm{H}_{2}\right)=(0.5-1) \times 10^{5} \mathrm{~cm}^{-3}$; the emission of the high- $J$ CO lines arises from a warmer $\left(T_{\text {kin }}=400-750 \mathrm{~K}\right)$, denser $\left(n\left(\mathrm{H}_{2}\right)=(0.5-1) \times 10^{6} \mathrm{~cm}^{-3}\right)$, lower column density $\left(N(\mathrm{CO})=1.5 \times 10^{16} \mathrm{~cm}^{-2}\right)$ gas component. Similarly, in the outflow cavity, two components are detected: the emission of the low- $J$ lines is dominated by a gas layer of column density $N(\mathrm{CO})=7 \times 10^{17} \mathrm{~cm}^{-2}$ at $T_{\text {kin }}=55-85 \mathrm{~K}$ and density in the range $(1-8) \times 10^{5} \mathrm{~cm}^{-3}$; the emission of the high- $J$ lines is dominated by a hot, denser gas layer with $T_{\text {kin }}=500-1500 \mathrm{~K}, n\left(\mathrm{H}_{2}\right)=(1-5) \times 10^{6} \mathrm{~cm}^{-3}$, and $N(\mathrm{CO})=6 \times 10^{16} \mathrm{~cm}^{-2} . \mathrm{A}$ temperature gradient as a function of the velocity is found in the high-excitation gas component. In the terminal bowshock HH377, we detect gas of moderate excitation, with a temperature in the range $T_{\text {kin }} \approx 400-500 \mathrm{~K}$, density $n\left(\mathrm{H}_{2}\right) \simeq(1-2) \times 10^{6} \mathrm{~cm}^{-3}$ and column density $N(\mathrm{CO})=10^{17} \mathrm{~cm}^{-2}$. The amounts of momentum carried away in the jet and in the entrained ambient medium are similar. Comparison with time-dependent shock models shows that the hot gas emission in the jet is well accounted for by a magnetized shock with an age of $220-740 \mathrm{yr}$ propagating at $20-30 \mathrm{~km} \mathrm{~s}^{-1}$ in a medium of density $n\left(\mathrm{H}_{2}\right)=(0.5-1) \times 10^{5} \mathrm{~cm}^{-3}$, consistent with that of the bulk material.

Conclusions. The CepE protostellar outflow appears to be a convincing case of jet bowshock driven outflow. Our observations trace the recent impact of the protostellar jet into the ambient cloud, produing a non-stationary magnetized shock, which drives the formation of an outflow cavity.

Key words. stars: formation - ISM: individual objects: Cep E - ISM: kinematics and dynamics - shock waves - infrared: ISM ISM: jets and outflows

\section{Introduction}

It is now well established that the formation of protostars powers fast jets surrounded by wide-angle winds that impact the highdensity parent cloud. These jets interact through shock fronts that compress and heat the ambient gas while driving the formation of low-velocity bipolar outflows. The low-excitation lines

\footnotetext{
* Appendices are available in electronic form at http://www . aanda.org
}

of CO have been the privileged tool of the study of protostellar outflows for decades (see e.g. Bachiller 1996). Many studies on $\mathrm{CO}$ outflow emission conducted from the ground have reported the presence of warm gas with a kinetic temperature in the range 50-200 K (see e.g. Hatchell et al. 1999a; Stark et al. 2004; van Kempen et al. 2006, 2009a,b; Gomez-Ruiz et al. 2013).

However, the atmospheric transmission at submillimeter and far-infrared wavelengths severely limits the $\mathrm{CO}$ transitions that can be observed from the ground above $J=7-6$. As a 
consequence, it is unclear whether any higher temperature component is present in the outflowing gas. In addition, the origin of the warm gas component is not well constrained and several processes have been proposed, such as outflow shocks, and irradiation processes associated with shocks or from the protostar (see e.g. van Kempen et al. 2009b). As suggested by Visser et al. (2012), the gas heating mechanism may depend on the evolutionary stage of the protostar itself.

This situation has changed with the advent of the Herschel Space Observatory ${ }^{1}$. For the first time, it has been become possible to observe in a systematic way the $\mathrm{CO}$ ladder up to very high energy levels $\left(J_{\text {up }}=16\right)$ at high spectral resolution. This allows a much more accurate determination of the physical conditions and the origin of the high-temperature gas in protostellar outflows. Recently, Yildiz et al. (2013) have carried out a systematic study of the high- $J$ lines of ${ }^{12} \mathrm{CO}$ and its isotopologues up to $J_{\text {up }}=10\left(E_{\text {up }}\right.$ up to $\left.300 \mathrm{~K}\right)$ towards a sample of 26 deeply embedded low-mass Class 0 and Class I young stellar objects as part of the Water In Star-forming regions with Herschel (WISH) key program (van Dishoeck et al. 2011). The aim was to constrain the physical characteristics (excitation, kinematics, column density) of the warm gas in low-mass protostellar envelopes and compare them with the colder gas traced by lower excitation lines. The authors have reported $\mathrm{CO}$ excitation temperatures in the range $60-130 \mathrm{~K}$. The outflow of the star-forming region L1157 is one of the very few outflows whose emission was investigated at high spectral resolution in the high-excitation lines of $\mathrm{CO}$, up to $J_{\text {up }}=16\left(E_{\text {up }}=751.8 \mathrm{~K}\right)$, as part of the Chemical HErschel Spectral Surveys (CHESS) key program (Ceccarelli et al. 2010). Lefloch et al. (2012) and Busquet et al. (2014) showed evidence for temperature stratification inside the outflow, with the presence of temperature components above $210 \mathrm{~K}$, which are best studied in the high-excitation transitions of CO $J_{\text {up }} \geq 10$.

The heterodyne receiver GREAT ${ }^{2}$ on board SOFIA is the only heterodyne instrument that permits observation of the highly excited $\mathrm{CO}$ transitions $J_{\text {up }}=11-22$, at an angular resolution of $10^{\prime \prime}-20^{\prime \prime}$, hence comparable to the ground-based submillimeter telescopes.

The intermediate-mass Class 0 source CepE-mm ( $L=$ $100 L_{\odot}$; Lefloch et al. 1996, Chini et al. 2001) in the Cepheus OB3 association at a distance of $730 \mathrm{pc}$ (Sargent 1977) drives an exceptionally powerful and luminous molecular outflow, which has long been studied in the rovibrational and pure rotational lines of $\mathrm{H}_{2}$ (Eisloeffel et al. 1996; Noriega-Crespo et al. $1998,2004,2014)$. Both the protostar and the outflow have been studied in the millimeter to far-infrared wavelength range since their discovery (Eislöffel et al. 1996; Lefloch et al. 1996; Noriega-Crespo et al. 1998, 2004b; Hatchell et al. 1999b; MoroMartin et al. 2001; Froebrich et al. 2003; Smith et al. 2003). Lefloch et al. (1996) reported the presence of high-velocity bullets of molecular gas in the outflow. The southern lobe is terminated by the Herbig-Haro object HH377, detected at optical wavelengths (Ayala et al. 2000).

Giannini et al. (2001) and Moro-Martin et al. (2001) analyzed the (unresolved) far-infrared emission lines of $\mathrm{CO}$ and $\mathrm{H}_{2} \mathrm{O}$ in Cep E, as observed with ISO. They found that the high- $J$ CO emission $\left(J_{\text {up }}=14-25\right)$ can be accounted for by an extended

\footnotetext{
1 Herschel is an ESA space observatory with science instruments provided by European-led Principal Investigator consortia and with important participation from NASA.

2 GREAT is a development by the MPI für Radioastronomie and the KOSMA/Universität zu Köln, in cooperation with the MPI für Sonnensystemforschung and the DLR Institut für Planetenforschung.
}

gas component with density and temperature in the range $4 \times$ $10^{4}-4 \times 10^{6} \mathrm{~cm}^{-3}$ ) and $200-1000 \mathrm{~K}$, respectively. Lefloch et al. (2011) reported the presence of water-rich shocked clumps of gas in the protostellar jet of Cep E, in which they estimated typical densities $n\left(\mathrm{H}_{2}\right) \simeq 10^{6} \mathrm{~cm}^{-3}$ and temperatures $T_{\text {kin }} \sim 200 \mathrm{~K}$ from the detection of the masing water line $3_{1,3}-2_{2,0}$ emission at $183.3 \mathrm{GHz}$. Recent observations of $\mathrm{HH} 377$ at high spectral resolution with GREAT/SOFIA by Gómez-Ruiz et al. (2012) showed that the emission of the CO $J=12-11$ and 13-12 lines in those bullets was consistent with gas at a temperatures higher than $100 \mathrm{~K}$ and density higher than $10^{4} \mathrm{~cm}^{-3}$. Interestingly, they discovered an additional high-velocity bullet in the high- $J$ line emission. However, the compact size of the outflow $\left(\sim 30^{\prime \prime}\right.$ in each lobe, see Figs. 1-2) with respect to the angular resolution of the observations $\left(20^{\prime \prime}\right)$ made it difficult to pinpoint the origin of the $\mathrm{CO}$ emission.

In this article, we report on a multi-transition study of the $\mathrm{CO}$ rotational ladder from millimeter to far-infrared wavelengths of the southern lobe of the Cep E-mm outflow. The data were collected with the IRAM 30 m telescope $(J=1-0)$ and Plateau de Bure interferometer (PdBI; $J=2-1$ ), the James Clerk Maxwell Telescope (JCMT; $J=3-2, J=4-3)$, Herschel $(J=$ $5-4,9-8,14-13)$, and SOFIA $(J=12-11,13-12,16-15)$. This is the most complete coverage of the $\mathrm{CO}$ ladder ever carried out at high spectral resolution towards a protostellar outflow. We used a map of the $J=2-1$ line obtained with the PdBI at $1^{\prime \prime}$ resolution to characterize the origin of the features detected with single-dish observations. The observations are described in Sect. 2. We describe in Sect. 3 the various components of the outflow and their specific spectral signatures, detected both with the PdBI and the single-dish telescopes: the protostellar jet, the bowshock HH377, and the outflow cavity. We present in Sect. 4 an analysis of the physical conditions in each outflow component. The properties of the jet and the origin of the high-excitation line emission are discussed in Sect. 5. Our conclusions are summarized in Sect. 6.

\section{Observations}

We describe below the CO line observations carried out with each of the following observatories: SOFIA, IRAM 30 m, IRAM PdBI, JCMT, and Herschel. Line intensities are expressed in units of antenna temperature corrected for atmospheric attenuation (for ground-based observations) $T_{\mathrm{A}}^{*}$. The observation and telescope parameters are summarized in Table 1 . The calibration uncertainty is within $10 \%$ in the millimeter bands $\left(J_{\text {up }}=1,2\right)$ and up to $20 \%$ in the submillimeter bands $\left(J_{\text {up }}=3,4,5,9,12\right.$, $13,14,16)$.

\subsection{SOFIA}

The observations in the Cep E star-forming region were conducted with the GREAT spectrometer (Heyminck et al. 2012). The position of the HH377 object, coinciding with the terminal bowshock of the outflow was targeted, with coordinates $\alpha(2000)=23^{\mathrm{h}} 03^{\mathrm{m}} 11^{\mathrm{s}} .6, \delta(2000)=61^{\circ} 42^{\prime} 03^{\prime \prime} .5$ (see Fig. 2 ). The receiver was tuned to the frequency of the CO lines $J=13-12$ at $1496.923 \mathrm{GHz}$ and $J=16-15$ at $1841.346 \mathrm{GHz}$, respectively, during the Cycle 1 SOFIA flight on 2 November 2013. The emission of the CO line $J=12-11$ at $1381.995 \mathrm{GHz}$ was observed towards the same position in July 2011 during Basic Science flights (Gomez-Ruiz et al. 2012).

The receiver was connected to a digital FFT spectrometer (Klein et al. 2012) providing a bandwidth of $1.5 \mathrm{GHz}$, 


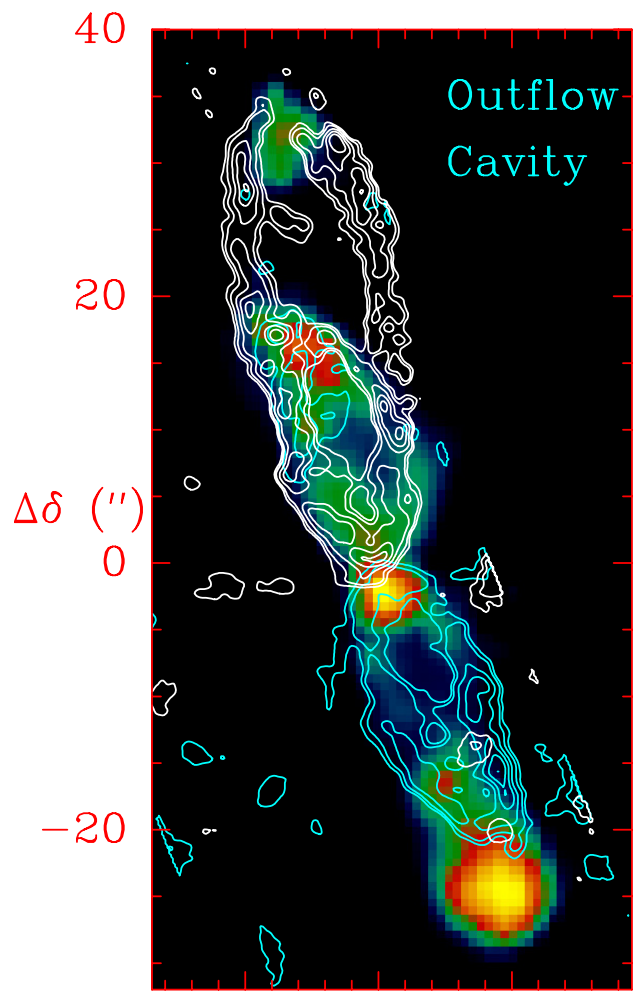

$10 \stackrel{0}{\Delta \alpha\left({ }^{\prime \prime}\right)}-10$

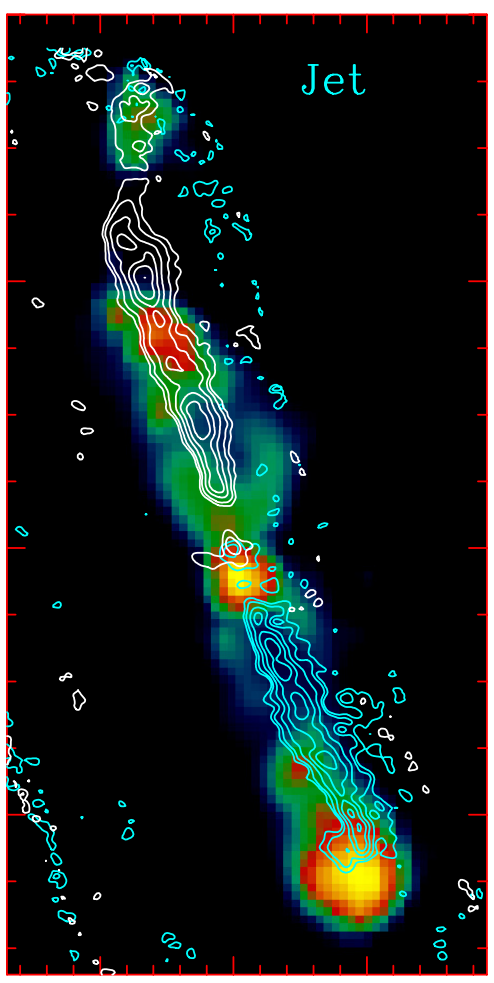

$10 \quad 0 \quad-10$

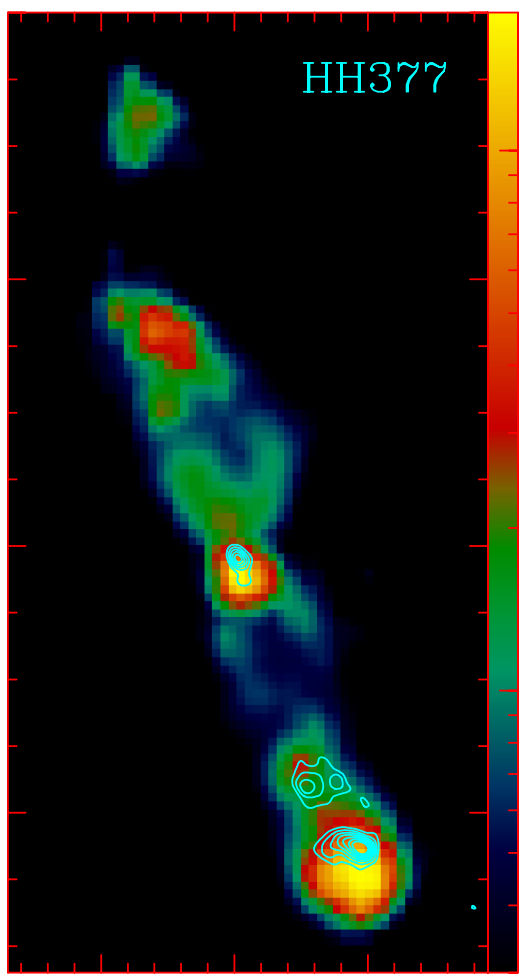

100

Fig. 1. Cep E outflow emission observed in the CO $J=2-1$ line with the PdBI at $1^{\prime \prime}$ resolution (contours) and with IRAC/Spitzer $\mathrm{H}_{2} 4.5 \mu \mathrm{m}$ line (color). Each panel shows the emission associated with each of the three outflow components identified: (left) outflow cavity walls emission, integrated between -15.2 and $-8.7 \mathrm{~km} \mathrm{~s}^{-1}$ in the southern lobe (blue contours) and between -2.2 and $-8.7 \mathrm{~km} \mathrm{~s}{ }^{-1}$ in the northern lobe (white contours); (center) jet emission integrated between -135 and $-110 \mathrm{~km} \mathrm{~s}^{-1}$ and between +40 and $+80 \mathrm{~km} \mathrm{~s} \mathrm{~s}^{-1}$ towards the southern (blue contours) and northern lobes (white contours), respectively; (right) HH377 bowshock emission integrated between -77 and -64 km s ${ }^{-1}$ (blue contours). First contour and contour interval are $20 \%$ and $10 \%$ of the peak intensity, respectively.

and resulting in the spectral resolutions given in Table 1. The observations were performed in double beam-switching mode, with an amplitude of $80^{\prime \prime}$ (or a throw of $160^{\prime \prime}$ ) at the position angle of $135^{\circ}$, and a phase time of $0.5 \mathrm{~s}$. The nominal focus position was updated regularly against temperature drifts of the telescope structure. The pointing was established with the optical guide cameras to an accuracy of $\sim 5^{\prime \prime}$. The data were calibrated with the KOSMA/GREAT calibrator (Guan et al. 2012), removing residual telluric lines, and further processed with the GILDAS/CLASS software ${ }^{3}$. We caution that the illumination of the telescope changed between Cycle 0 and Cycle 1. The telescope efficiencies have been recently revised. We adopted the values displayed on the SOFIA website ${ }^{4}$ (see also Table 1).

\subsection{IRAM $30 \mathrm{~m}$}

The emission of the Cep E outflow in the CO transitions $J=$ $1-0$ at $115.271 \mathrm{GHz}, J=2-1$ at $230.538 \mathrm{GHz}$ and ${ }^{13} \mathrm{CO} J=$ 2-1 at $220.399 \mathrm{GHz}$ was observed with the IRAM $30 \mathrm{~m}$ telescope in January 2011. Deep integrations were carried out using the EMIR receivers towards HH377, targeting the position $\alpha(2000)=23^{\mathrm{h}} 03^{\mathrm{m}} 11^{\prime \prime} .1 \delta(2000)=+61^{\circ} 42^{\prime} 08^{\prime \prime}$. The observations were carried out in Wobbler Switching Mode, with a throw of $3^{\prime}$, and using the broadband EMIR receivers. We used the FTS

\footnotetext{
3 http://www .iram. fr/IRAMFR/GILDAS

4 http://www3.mpifr-bonn.mpg.de/div/submmtech/ heterodyne/great/GREAT_calibration.html
}

spectrometers in its $200 \mathrm{kHz}$ resolution mode, corresponding to velocity resolutions of $0.6 \mathrm{~km} \mathrm{~s}^{-1}$, for the $J=1-0$ observations; the WILMA spectrometer with $2 \mathrm{MHz}$ resolution was used for the observations of the ${ }^{12} \mathrm{CO}$ and ${ }^{13} \mathrm{CO} J=2-1$, providing a velocity resolution of about $2.7 \mathrm{~km} \mathrm{~s}^{-1}$. The data were processed using the GILDAS/CLASS software.

We obtained a fully sampled map of $2^{\prime} \times 2^{\prime}$ centered on the protostellar source CepE-mm with the receiver A230 in July 1996, using the On-The-Fly mode. The map of the CO $J=$ 2-1 emission integrated in channels of $3.25 \mathrm{~km} \mathrm{~s}^{-1}$ is displayed in Figs. A.1, A.2. This permitted us to obtain the spectrum of the CO $J=2-1$ line at $230.538 \mathrm{GHz}$ towards the same position as the $J=1-0$ observations in a beam of $20^{\prime \prime}$ size (HPBW), hence comparable to the resolution of the Herschel CO $J=9-8$ and the IRAM CO $J=1-0$ observations. We present in Fig. 3 the spectrum of the CO $J=2-1$ emission in a beam of $20^{\prime \prime}$ halfpower beamwidth (HPBW). We adopted the forward and beam efficiency values $F_{\text {eff }}$ and $B_{\text {eff }}$, as monitored by IRAM $^{5}$ (see Table 1).

\section{3. $P d B I$}

The $J=2-1$ line emission in the CepE protostellar outflow was observed with the five-antenna array of the Plateau de Bure Interferometer in March 1997 in configurations C and D,

http://www.iram.fr 


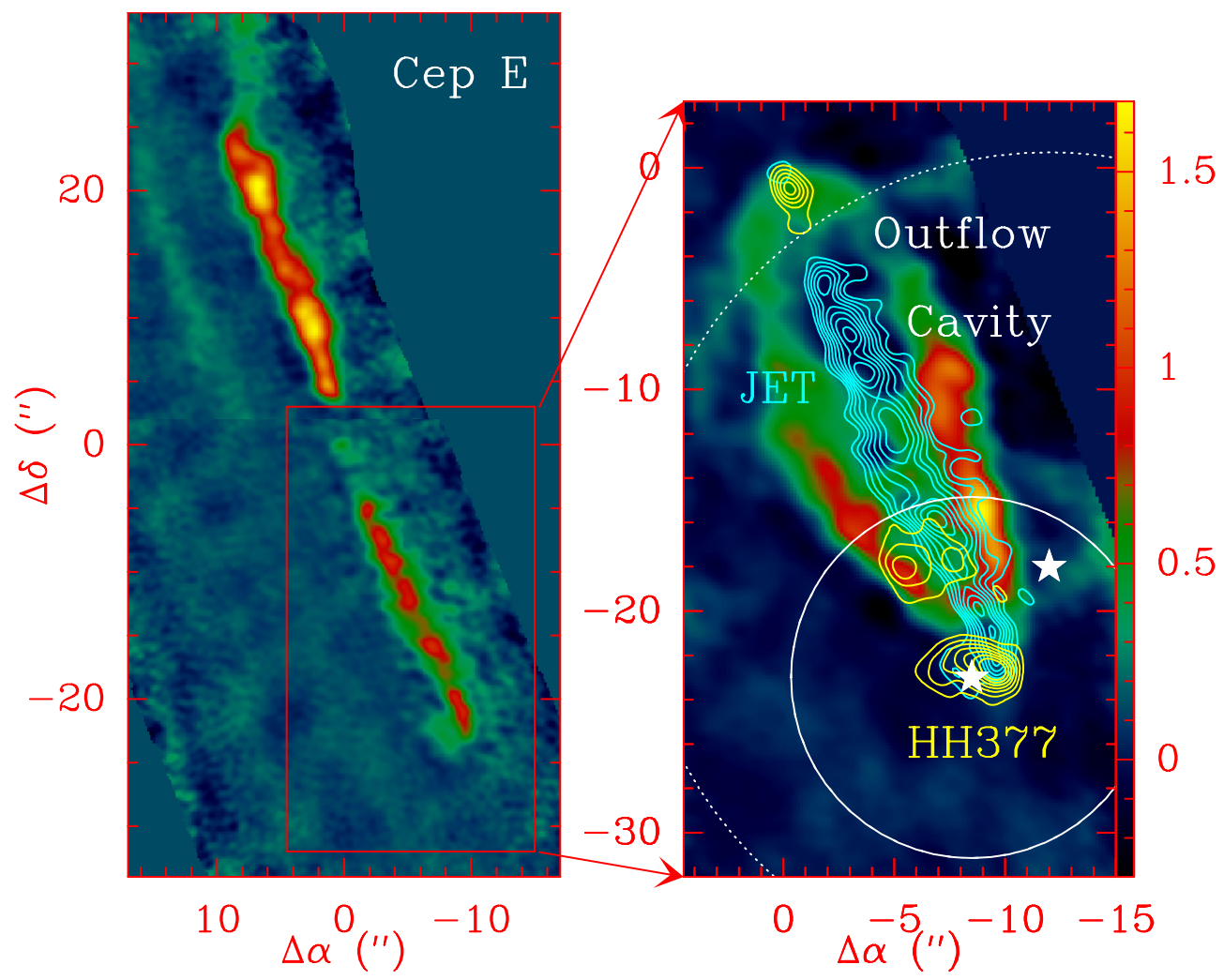

Fig. 2. Left: $\mathrm{CO} J=2-1$ velocity-integrated emission of the protostellar jet observed with the PdBI. The emission is integrated between -135 and $-110 \mathrm{~km} \mathrm{~s}^{-1}$ and between +40 and $+80 \mathrm{~km} \mathrm{~s}^{-1}$ towards the southern and northern lobes, respectively. Right: the three kinematic components in the southern lobe of the Cepheus E outflow as seen by the PdBI at $1^{\prime \prime}$ resolution in the CO $J=2-1$ line: a) outflow cavity emission between -15 and $-12 \mathrm{~km} \mathrm{~s}^{-1}$ (color); b) HH377 bowshock emission between -77 and $-64 \mathrm{~km} \mathrm{~s}^{-1}$ (yellow contours); c) jet emission between -135 and $-110 \mathrm{~km} \mathrm{~s}^{-1}$ (blue contours). First contour and contour interval are $20 \%$ and $10 \%$ of the peak intensity, respectively. The white stars mark the location of the positions observed with SOFIA $\left(-8.5^{\prime \prime},-23^{\prime \prime}\right)$ and Herschel (HSO), IRAM, and JCMT $\left(-12^{\prime \prime},-18^{\prime \prime}\right)$. The beam width at half-power of SOFIA in the CO $J=16-15$ and of HIFI in the $J=5-4$ are drawn by a solid and a dotted circle, respectively.

Table 1. CO line observation parameters and velocity-integrated line flux of the outflow components: jet, shock, outflow cavity.

\begin{tabular}{|c|c|c|c|c|c|c|c|c|c|c|c|}
\hline $\begin{array}{l}\mathrm{CO} \\
\text { transition }\end{array}$ & $\begin{array}{r}v \\
(\mathrm{GHz}) \\
\end{array}$ & $\begin{array}{r}E_{\mathrm{u}} \\
(\mathrm{K}) \\
\end{array}$ & Telescope & $\begin{array}{c}H P B W \\
(")\end{array}$ & $F_{\text {eff }}$ & $B_{\text {eff }}$ & $\begin{array}{c}\Delta v \\
\left(\mathrm{~km} \mathrm{~s}^{-1}\right) \\
\end{array}$ & $\begin{array}{c}\mathrm{rms} \\
\left(T_{\mathrm{mb}} m K\right) \\
\end{array}$ & $\begin{array}{c}\text { Jet } \\
\mathrm{K} \mathrm{km} \mathrm{s}^{-1} \\
\end{array}$ & $\begin{array}{r}\text { Shock } \\
\mathrm{K} \mathrm{km} \mathrm{s}^{-1} \\
\end{array}$ & $\begin{array}{r}\text { Outflow cavity } \\
\mathrm{K} \mathrm{km} \mathrm{s}^{-1} \\
\end{array}$ \\
\hline $1-0$ & 115.2713 & 5.5 & IRAM & 21.4 & 0.95 & 0.78 & 0.51 & 11 & 1.38 & - & 17.0 \\
\hline $2-1$ & 230.5380 & 16.6 & IRAM & 20.0 & 0.92 & 0.45 & 1.30 & 50 & 6.14 & - & 67.8 \\
\hline $3-2$ & 345.7959 & 33.2 & JCMT & 20.0 & 1.0 & 0.67 & 0.54 & 77 & 9.38 & - & 101.8 \\
\hline $4-3$ & 461.0408 & 55.3 & JCMT & 10.7 & 1.0 & 0.45 & 0.61 & 509 & 23.31 & - & 252.8 \\
\hline $5-4$ & 576.2679 & 83.0 & HIFI & 37.4 & 0.96 & 0.75 & 0.14 & 7 & 5.21 & 3.4 & 33.9 \\
\hline $9-8$ & 1036.9124 & 248.9 & HIFI & 20.5 & 0.96 & 0.74 & 0.14 & 11 & 7.28 & 12.5 & 42.1 \\
\hline $12-11$ & 1381.9950 & 431.3 & SOFIA & 21.7 & 0.95 & 0.55 & 0.04 & 81 & 2.33 & 8.1 & 13.7 \\
\hline $13-12$ & 1496.9229 & 503.1 & SOFIA & 17.7 & 0.97 & 0.67 & 0.037 & 48 & 3.05 & 12.2 & 20.5 \\
\hline $14-13$ & 1611.7935 & 580.5 & HIFI & 13.2 & 0.95 & 0.71 & 0.19 & 55 & 3.32 & 10.5 & 18.2 \\
\hline $16-15$ & 1841.3455 & 751.7 & SOFIA & 14.5 & 0.97 & 0.65 & 0.030 & 58 & 2.83 & 13.9 & 21.0 \\
\hline \multicolumn{12}{|l|}{${ }^{13} \mathrm{CO}$} \\
\hline $2-1$ & 220.3987 & 15.9 & IRAM & 11.2 & 0.92 & 0.61 & 2.7 & 8 & - & - & 5.3 \\
\hline $5-4$ & 550.9263 & 79.3 & HIFI & 38.5 & 0.95 & 0.75 & 2.5 & 11 & - & - & 0.68 \\
\hline
\end{tabular}

Notes. The root-mean square (rms) are expressed in units of main-beam brightness temperature per velocity interval of $1 \mathrm{~km} \mathrm{~s}^{-1}$. Fluxes are integrated between -140 and $-100 \mathrm{~km} \mathrm{~s}^{-1},-90$ and $-50 \mathrm{~km} \mathrm{~s}^{-1}$, and -50 and $-17 \mathrm{~km} \mathrm{~s}^{-1}$ for the jet, HH377, and the outflow cavity, respectively. They are expressed in units of main-beam brightness temperature.

and again in January and February 1998 in configurations A and B. In order to cover both lobes of the outflow, a mosaic of seven fields overlapping at half power was observed, covering an area of approximately $24^{\prime \prime} \times 90^{\prime \prime}$. The size of the synthesized beam is $1.07^{\prime \prime} \times 0.87^{\prime \prime}$ and makes a PA of $73 \mathrm{deg}$. We used a spectral correlator configured to give 128 channels of $2.5 \mathrm{MHz}$ $\left(3.25 \mathrm{~km} \mathrm{~s}^{-1}\right.$ at the frequency of the $\mathrm{CO} \mathrm{J}=2-1$ line), allowing coverage of the full velocity range of outflow emission. Calibration was carried out following standard procedures, using the GILDAS/CLIC software ${ }^{6}$.

6 http://www.iram.fr/GILDAS/ 
B. Lefloch et al.: CO emission in the Cep E outflow: the outflow cavity, the bowshock, and the jet

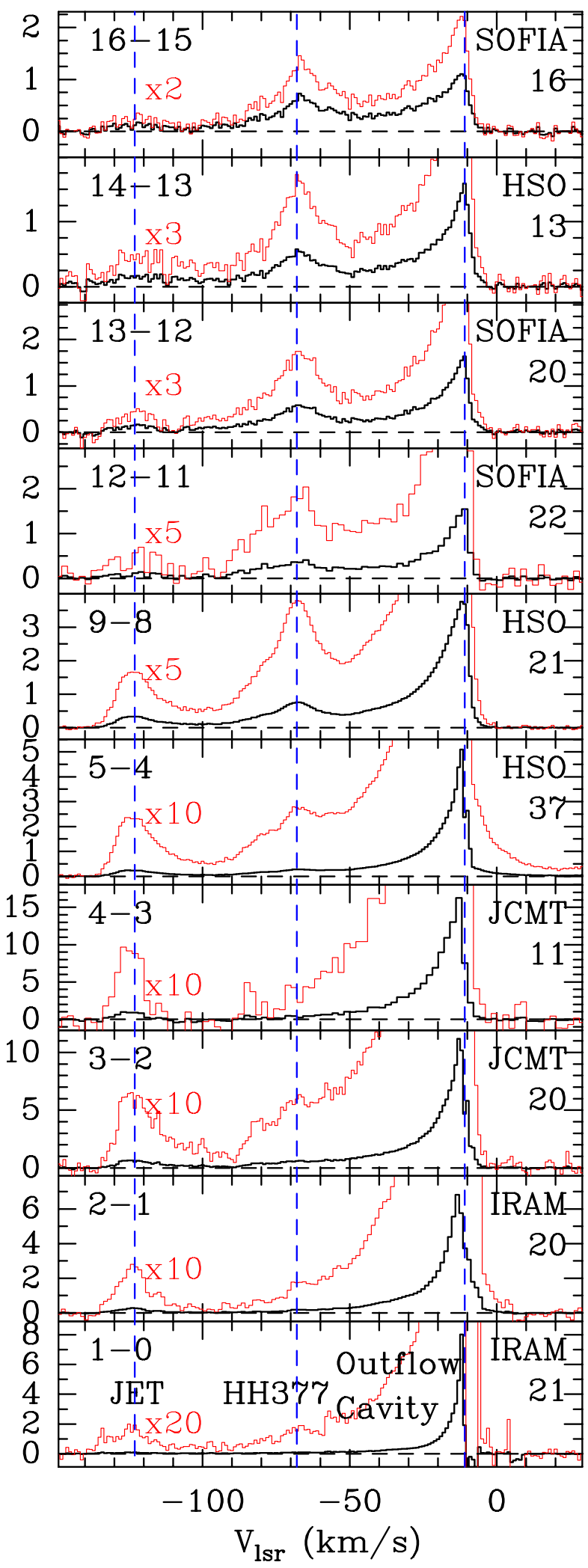

Fig. 3. Montage of all the CO transitions observed towards HH377. Line profiles are drawn in thick black. Spectra enhanced with a magnifying factor in order to enhance the high-velocity emission are drawn in thin red. Fluxes are expressed in units of antenna temperature $T_{\mathrm{A}}^{*}$, corrected for atmospheric absorption (for ground-based observations). The ambient cloud velocity $\left(v_{\mathrm{lsr}}=-10.9 \mathrm{~km} \mathrm{~s}^{-1}\right)$, the peak velocity of the protostellar jet $\left(v=-125 \mathrm{~km} \mathrm{~s}^{-1}\right)$, and the bowshock HH377 $\left(v=-68 \mathrm{~km} \mathrm{~s}^{-1}\right)$ are marked by dashed blue lines. On the right hand side of each panel we have indicated the observatory and the HPBW in arcsec.
Our IRAM 30 m map was used to provide the short spacings for our PdBI map. We checked that the flux of the jet (both blueand redshifted components) and of HH377 were unchanged to better than $97 \%$ and $87 \%$, respectively, when including the short spacings, i.e. they were fully recovered by the interferometer. By comparison, $52 \%$ of the flux from the southern outflow cavity was recovered by the interferometer.

We present here the interferometric data uncorrected for the missing flux, which therefore affects only the outflow cavity, with the motivation of highlighting the structure of the outflow itself, as can be seen in Figs. 1, 2. We note that the global properties of the outflow cavity (mass, density, temperature, column density, momentum) were all derived from the single-dish observations. Hence, the flux filtered out by the interferometer does not affect the qualitative analysis presented here.

\subsection{JCMT}

Observations of the CO lines $J=3-2345.796 \mathrm{GHz}$ and $J=$ 4-3 461.041 GHz were carried out at the JCMT in June 1997. The outflow emission was mapped at $6^{\prime \prime}$ sampling in the $J=$ 3-2 line over an area $42^{\prime \prime} \times 60^{\prime \prime}$, centered on the position of the protostar Cep E-mm. The map of the CO $J=3-2$ emission integrated in channels of $3.25 \mathrm{~km} \mathrm{~s}^{-1}$ is shown in Figs. A.3-A.4. At the frequency of the $J=3-2$ line, the HPBW of the telescope is $\approx 15^{\prime \prime}$, and the main-beam efficiency of the telescope is 0.67 . This map permitted us to obtain the spectrum of the CO $J=$ 3-2 line in a beam of 20" (HPBW). A deep integration was performed towards the position $\alpha(2000)=23^{\mathrm{h}} 03^{\mathrm{m}} 11.1^{\mathrm{s}} \delta(2000)=$ $+61^{\circ} 42^{\prime} 08.0^{\prime \prime}$, at offset position $\left(-12^{\prime \prime}-18^{\prime \prime}\right)$ with respect to the protostar Cep E-mm. The observations were carried out in Wobbler Switching Mode with a throw of $3^{\prime}$ using receiver RxB3 and the Digital Autocorrelating Spectrometer (DAS) in its $625 \mathrm{kHz}$ resolution mode corresponding to a velocity resolution of $\approx 0.54 \mathrm{~km} \mathrm{~s}^{-1}$. The data were converted from FITS to GILDAS format, and subsequently reduced with CLASS.

\subsection{Herschel}

The emission in the CO transitions $J=5-4576.268 \mathrm{GHz}, J=$ 9-8 1036.912 GHz, $J=14-131611.794 \mathrm{GHz}$, and ${ }^{13} \mathrm{CO} J=$ 5-4 550.296 GHz was observed with the HIFI spectrometer on board the Herschel Space Observatory on 30 January and 2 February 2013, in the course of the program SPECSO, dedicated to spectroscopy of outflow shocks (PIs: Lefloch \& Benedettini). The position targeted was $\alpha(2000)=23^{\mathrm{h}} 03^{\mathrm{m}} 11.1^{\mathrm{s}}$ $\delta(2000)=+61^{\circ} 42^{\prime} 08.0^{\prime \prime}$, which is offset from the SOFIA position by $-3.5^{\prime \prime}$ in RA and $-5^{\prime \prime}$ in declination (see Fig. 2). The Obs_Id of the $\mathrm{J}=5-4,9-8,14-13$ observations are 1342262584, 1342262801,1342262555 , respectively.

Observations were carried out in double beam switching mode with a throw of $3 \mathrm{arcmin}$. The receiver was tuned in double sideband and the Wide Band Spectrometer was used, providing a spectral resolution of $1.1 \mathrm{MHz}$, which was subsequently degraded to reach a final velocity resolution of $0.5 \mathrm{~km} \mathrm{~s}^{-1}$. The telescope parameters $\left(F_{\text {eff }}, B_{\text {eff }}\right.$, HPBW) were adopted from Roelfsema et al. (2012; see Table 1). The data were processed with the ESA-supported package Herschel interactive processing environment ( $\left.\mathrm{HIPE}^{7}\right)$. FITS files from level 2 data were then

HIPE is a joint development by the Herschel Science Ground Segment Consortium, consisting of ESA, the NASA Herschel Science Center, and the HIFI, PACS, and SPIRE consortia. 
created and transformed into GILDAS format for baseline subtraction and subsequent data analysis.

\section{Results}

Our large-scale maps of the CO $J=2-1$ and 3-2 emission reveal a double outflow structure centered on the protostellar core (Figs. A.1-A.4): the compact ( size $\sim 1^{\prime}$ ), collimated highvelocity outflow, oriented in the southwest-northeast direction, and an extended (size $\sim 3^{\prime}$ ), less collimated, lower velocity outflow, propagating in the southeast-northwest direction. The relative postion angles of both outflows differ by $52 \mathrm{deg}$. We do not find evidence for source multiplicity down to $1^{\prime \prime}$ (730 AU) in our PdBI map. This double outflow structure was reported for the first time by Ladd \& Hodapp (1997). These authors noticed that the high-velocity outflow is associated with strong line emission from vibrationally excited molecular hydrogen, while the large-scale outflow structure shows no associated molecular hydrogen emission. In this work, we present a detailed study of the collimated, high-velocity outflow, hereafter "the outflow".

\subsection{Multiple outflow components}

Our CO $J=2-1$ interferometric observations reveal three spatial components in the protostellar outflow: the molecular jet, the outflow cavity, and the terminal shock HH377. In Fig. 1, we superpose the emission of each outflow component on an IRAC/Spitzer (channel 2) image at $2^{\prime \prime}$ resolution of the $\mathrm{H}_{2}$ emission (Noriega-Crespo et al. 2004). IRAC channel 2 at $4.5 \mu \mathrm{m}$ is an excellent tracer of the $\mathrm{H}_{2}$ pure rotational emission, since three of the brightest lines, $0-0 \mathrm{~S}(11) 4.18 \mu \mathrm{m}, 0-0 \mathrm{~S}(10) 4.40 \mu \mathrm{m}$, and $0-0 \mathrm{~S}(9) 4.18 \mu \mathrm{m}$ fall within its passband. Figure 2 shows a superposition of the emission of the three components, contributing to the emission of the southern outflow lobe. A montage of all the CO transitions observed towards Cep E with IRAM 30 m, JCMT, Herschel, and SOFIA is presented in Fig. 3. Each of the components identified in the PdBI map appears to have a specific spectral signature.

The molecular jet powered by the protostar is detected between $v_{\mathrm{lsr}}=-140$ and $-100 \mathrm{~km} \mathrm{~s}^{-1}$ (Fig. 3). The emission is detected as a line typically $15 \mathrm{~km} \mathrm{~s}^{-1}$ wide and well separated from the bipolar outflow wing emission. Our PdBI map shows that the jet is approximately $21^{\prime \prime}$ long $\left(1.5 \times 10^{4}\right.$ AU, beamdeconvolved), with a transverse size of 1 .'7, or 1200 AU (beamdeconvolved). The jet emission terminates at the offset position $\left(-9^{\prime \prime} ;-23^{\prime \prime}\right)$, at 0.5 upstream of the nominal position of $\mathrm{HH} 377$ (Fig. 1). There is no evidence of highly excited $\mathrm{H}_{2}$ emission, as traced by IRAC, along the jet.

The blueshifted wing of the bipolar outflow is detected from the ambient cloud velocity $\left(v_{\mathrm{lsr}}=-10.9 \mathrm{~km} \mathrm{~s}^{-1}\right.$; Lefloch et al. 1996) up to $-100 \mathrm{~km} \mathrm{~s}^{-1}$ in the low- $J$ transitions of CO (Figs. 1-3). In the PdBI map, the emission arises from the outflow cavity walls, which appear to tightly surround the protostellar jet. The emission is distributed into two imbricated shells, which provides evidence for multiple ejection. The bulk of the emission is detected up to $10 \mathrm{~km} \mathrm{~s}^{-1}$ from the ambient cloud velocity (Fig. 3). Comparison with the IRAC/Spitzer image shows an excellent match between the $\mathrm{CO} J=2-1$ and $\mathrm{H}_{2}$ line emission from the outflow cavity walls, as traced by IRAC2 and the PdBI. The outflow cavity can be modeled by an ellipse with a major (minor) axis of $22^{\prime \prime}\left(10^{\prime \prime}\right)$ (beam-deconvolved) with a PA of $70 \mathrm{deg}$; see Fig. 1. In what follows, we refer to the outflow cavity as the gas that is contained within the cavity walls, which is not part of the molecular jet, such as detected in our PdBI map.
The terminal shock HH377 is seen downstream of the molecular jet, close to the offset position $\left(-9^{\prime \prime},-23^{\prime \prime}, 5\right)$; it is detected in the velocity range $-90 ;-50 \mathrm{~km} \mathrm{~s}^{-1}$, i.e. at intermediate velocities between the outflow cavity and the jet. At the PdBI, in CO $J=2-1$, it is detected as a bright component downstream of the molecular jet, with a typical size of $\approx 4^{\prime \prime}$ (beamdeconvolved). The $\mathrm{CO} J=2-1$ emission region coincides with the rear of HH377, as observed with IRAC/Spitzer (Fig. 1). This suggests that the CO $J=2-1$ line traces the post-shocked gas layer, whereas the pure $\mathrm{H}_{2}$ lines trace the high-excitation gas in the shock.

In the same velocity range, two weaker components are detected in addition to HH377. Both display a compact size of a few arcsec. The first coincides with the clump of shocked gas reported by Lefloch et al. (2011) in the inner envelope of the protostar; the second coincides with the infrared knot BI reported by Gomez-Ruiz et al. (2012).

The emission from HH377 appears as a bright "bump" in the highest excitation lines of CO $\left(J_{\text {up }}=16\right)$, whose intensity is similar to that of the entrained gas at $v=-20 \mathrm{~km} \mathrm{~s}^{-1}$. The intensity of the $\mathrm{HH} 377$ emission decreases in the lower-excitation lines of $\mathrm{CO}$, and appears to be fully dominated by the entrained gas in the transitions $J_{\text {up }} \leq 4$ (Fig. 2). This is consistent with highly-excited emission from a small region.

\subsection{Dynamical age}

Noriega-Crespo et al. (2014) have measured the proper motions in the jet of Cep E using IRAC images containing mostly a mix of emission of several $\mathrm{H}_{2}$ lines obtained over a period of 6 years. They derived tangential velocities of the northern and southern outflow lobes of $62 \pm 29$ and $94 \pm 26 \mathrm{~km} \mathrm{~s}^{-1}$, respectively.

Adopting the value measured in our $\mathrm{CO}$ observations (see above) as jet radial velocity, we derive both the jet velocity $V_{\mathrm{j}}$ and the jet inclination with respect to the line of sight. We find a jet velocity of $+107 \mathrm{~km} \mathrm{~s}^{-1}$ and $-148 \mathrm{~km} \mathrm{~s}^{-1}$ ) in the northern and southern lobes, respectively. We find in a consistent way that the jet makes the same angle $\Phi=40 \mathrm{deg}$ with respect to the line of sight in both outflow lobes. The difference in the jet velocity could arise from a density gradient in the cloud, with a higher density towards the north.

We have estimated the dynamical age of the terminal bowshock HH377, located at $26^{\prime \prime}(0.092 \mathrm{pc})$ from the protostar, as $\tau_{\text {dyn }}=d / V_{\mathrm{j}} \sin \Phi$ where $d$ is the distance of HH377 to the location of the protostar; it comes $\tau_{\text {dyn }}=960 \mathrm{yr}$. The small size of both outflow lobes and the young dynamical age suggest that the jet is probably at an early evolutionary stage.

\section{Outflow physical conditions}

\subsection{Methodology}

The determination of the physical conditions in the outflow is made difficult by the presence of the second large-scale outflow (see above). As discussed in Sect. 3, the CO emission of both outflows overlap between +2 and $-17 \mathrm{~km} \mathrm{~s}^{-1}$ in the central $30^{\prime \prime}$ around the protostar (Figs. A.1 to A.4). We note that the CO $J=2-1$ emission from the large-scale outflow is detected up to $-30 \mathrm{~km} \mathrm{~s}^{-1}$. Therefore, it is difficult to disentangle the respective contributions of both outflows to the entrained gas (the outflow cavity) in the central $30^{\prime \prime}$ around the protostar. The absence of $\mathrm{H}_{2}$ emission associated with the large-scale outflow suggests, however, low excitation in the latter, so that the contamination most likely affects only the low- $J$ transitions. In order 
B. Lefloch et al.: CO emission in the Cep E outflow: the outflow cavity, the bowshock, and the jet

Table 2. CO line velocity-integrated flux of the outflow components: jet, shock, outflow cavity.

\begin{tabular}{lrccccccrr}
\hline \hline $\begin{array}{l}\text { CO } \\
\text { transition }\end{array}$ & Telescope & $\begin{array}{c}H P B W \\
(")\end{array}$ & $\begin{array}{c}\mathrm{rms} \\
\mathrm{K} \mathrm{km} \mathrm{s}^{-1}\end{array}$ & $f f_{\text {jet }}$ & $\begin{array}{c}\text { Jet } \\
\mathrm{K} \mathrm{km} \mathrm{s}^{-1}\end{array}$ & $f f_{\text {shock }}$ & $\begin{array}{c}\text { Shock } \\
\mathrm{K} \mathrm{km} \mathrm{s}^{-1}\end{array}$ & $f f_{\text {cavity }}$ & $\begin{array}{r}\text { Outflow cavity } \\
\mathrm{K} \mathrm{km} \mathrm{s}^{-1}\end{array}$ \\
\hline $1-0$ & IRAM & 21.4 & 11 & 0.063 & 21.9 & - & - & 0.20 & 85 \\
$2-1$ & IRAM & 20.0 & 50 & 0.063 & 97.4 & - & - & 0.20 & 340 \\
$3-2$ & JCMT & 20.0 & 77 & 0.063 & 148.9 & - & - & 0.20 & 510 \\
$4-3$ & JCMT & 10.7 & 509 & 0.101 & 230.7 & - & - & 0.43 & 588 \\
$5-4$ & HIFI & 37.4 & 7 & 0.028 & 186.1 & 0.013 & 268 & 0.06 & 565 \\
$9-8$ & HIFI & 20.5 & 11 & 0.093 & 78.3 & 0.042 & 297 & 0.18 & 234 \\
$12-11$ & SOFIA & 21.7 & 81 & 0.050 & 43.6 & 0.028 & 290 & 0.14 & 98 \\
$13-12$ & SOFIA & 17.7 & 48 & 0.074 & 43.6 & 0.042 & 290 & 0.18 & 146 \\
$14-13$ & HIFI & 13.2 & 55 & 0.091 & 36.5 & 0.043 & 242 & 0.18 & 101 \\
$16-15$ & SOFIA & 14.5 & 58 & 0.090 & 31.4 & 0.063 & 220 & 0.14 & 150 \\
\hline
\end{tabular}

Notes. Fluxes are integrated between -140 and $-100 \mathrm{~km} \mathrm{~s}^{-1},-90$ and $-50 \mathrm{~km} \mathrm{~s}^{-1}$, and -50 and $-17 \mathrm{~km} \mathrm{~s}^{-1}$ for the jet, HH377, and the outflow cavity, respectively. Fluxes and rms are expressed in units of brightness temperature, corrected for the coupling between the source and the telescope main-beam.

Table 3. Physical conditions $\left(n\left(\mathrm{H}_{2}\right), T_{\text {kin }}, N(\mathrm{CO})\right)$ in the outflow cavity as a function of velocity between -14 and $-50 \mathrm{~km} \mathrm{~s}{ }^{-1}$, as derived from our large velocity gradient analysis of the velocity-integrated $\mathrm{CO}$ line fluxes.

\begin{tabular}{lrrrccccc}
\hline \hline Vel. range $\left(\mathrm{km} \mathrm{s}^{-1}\right)$ & & $-14 ;-17$ & $-17 ;-20$ & $-20 ;-25$ & $-25 ;-30$ & $-30 ;-35$ & $-35 ;-40$ & $-40 ;-50$ \\
\hline Low-excitation & $T_{\text {kin }}(\mathrm{K})$ & 85 & 75 & 75 & 70 & 65 & 65 & 62 \\
& $n\left(\mathrm{H}_{2}\right)\left(\mathrm{cm}^{-3}\right)$ & $0.8(5)$ & $2(5)$ & $1(5)$ & $2.7(5)$ & $4(5)$ & $6(5)$ & $8(5)$ \\
& $N(\mathrm{CO})\left(\mathrm{cm}^{-2}\right)$ & $2.0(17)$ & $1.0(17)$ & $8.5(16)$ & $5(16)$ & $3.5(16)$ & $2.5(16)$ & $3(16)$ \\
& $\tau_{54}^{12}$ & 2.9 & 1.5 & 0.90 & 0.50 & 0.35 & 0.25 & 0.15 \\
& $\tau_{21}^{12}$ & 0.9 & 0.50 & 0.27 & 0.18 & 0.13 & 0.09 & 0.06 \\
High-excitation & $T(\mathrm{~K})$ & $750-1200$ & $700-1200$ & $700-1100$ & $700-1100$ & $850-1200$ & $800-1500$ & $1000-1500$ \\
& $n\left(\mathrm{H}_{2}\right)\left(\mathrm{cm}^{-3}\right)$ & $(1-2)(6)$ & $(1-3)(6)$ & $(1-5)(6)$ & $(1-5)(6)$ & $(1-5)(6)$ & $(1-3)(6)$ & $(1-3)(6)$ \\
& $N(\mathrm{CO})\left(\mathrm{cm}^{-2}\right)$ & $8(15)$ & $6.5(15)$ & $9(15)$ & $7(15)$ & $6(15)$ & $4.5(15)$ & $8.5(15)$ \\
\hline
\end{tabular}

Notes. The optical depths predicted by our modeling of the low-excitation gas component for the $J=2-1$ and $5-4$ lines are given. The velocity interval of integration is given in the table.

to accurately derive the excitation conditions in the $\mathrm{CO}$ cavity gas, we have used the line fluxes integrated between -50 and $-17 \mathrm{~km} \mathrm{~s}^{-1}$, outside the velocity interval in which the outflows overlap.

The $\mathrm{CO}$ gas excitation temperature and column density were first derived from a simple rotational diagram analysis of the line fluxes, after correcting the intensities for the main-beam filling factor (see Goldsmith \& Langer 1999). The CO fluxes from the molecular jet and the outflow cavity emission were obtained from direct integration in the corresponding velocity range (see Table 2). The emission from the shock was obtained after subtracting the contribution of the entrained gas. As the shock emission is dominated by the entrained gas emission in the transitions $J_{\text {up }}=1-4$, only the emission in the transitions $\left(J_{\text {up }} \geq 5\right)$ is reported here and taken into account in the analysis.

For each component of the outflow (jet, HH377, cavity), we estimated the solid angle of the emission encompassed by the telescope beam at the frequency of each CO transition, from the intersection between the half-power contour of the $J=2-1$ emission mapped at the PdBI and the telescope main-beam modeled by a disk of diameter equal to the HPBW, centered at the targeted position. We then derived the corresponding beam filling factor from the ratio of the source solid angle to the telescope main beam in order to estimate the intrinsic brightness temperature of the three outflow components in each $\mathrm{CO}$ transition. The results of this procedure were successfully compared to the value obtained from a $\chi^{2}$ minimization of the CO line emission modeled with a radiative transfer code in the large velocity gradient approximation, as explained in Sect. 4.3; we feel confident that the procedure is valid. The integrated intensities are indicated in Tables 1 and 2, while the results are given in Table 3 .

\subsection{Line opacity}

We used the ${ }^{13} \mathrm{CO}$ lines $J=2-1$ and $5-4$ in order to constrain the opacity of the transitions of the main isotopologue in the outflow. The spectra of the ${ }^{13} \mathrm{CO}$ transitions are presented in the bottom panels of Fig. 4. The line profiles peak at ambient velocity $v_{\text {lsr }}=-10.9 \mathrm{~km} \mathrm{~s}^{-1}$. The linewidth is $\Delta v \simeq 2.7 \mathrm{~km} \mathrm{~s}^{-1}$. Emission is detected at the $3 \sigma$ level in the entrained gas up to $-50 \mathrm{~km} \mathrm{~s}^{-1}$. We note that the absorption features detected between -5 and $0 \mathrm{~km} \mathrm{~s}^{-1}$ in the $J=2-1$ spectrum are caused by contamination from the reference position adopted in the observations (see Sect. 2.2).

The ratio of the ${ }^{12} \mathrm{CO}$ to ${ }^{13} \mathrm{CO}$ line intensities is shown in the top panel of Fig. 4. A similar behavior is observed for both transitions. The ratio is strongly peaked at the ambient cloud velocity with values $\approx 0.4$ and 0.26 for the $J=2-1$ and $J=5-4$, respectively. These values refer to the ambient cloud molecular gas, however, and not to the entrained gas of the outflow. As the $J=5-4$ line ratio is sampled at $1 \mathrm{~km} \mathrm{~s}^{-1}$ resolution, it appears much more strongly peaked than the $J=2-1$ data, which are sampled at $3 \mathrm{~km} \mathrm{~s}^{-1}$ only. At $v=-14 \mathrm{~km} \mathrm{~s}^{-1}$ and $-17 \mathrm{~km} \mathrm{~s}^{-1}$, we measure a ratio of 0.10 and 0.032 for the $J=2-1$, respectively, and 0.047 and 0.032 for the $J=5-4$. Adopting a standard abundance ratio $\left[{ }^{12} \mathrm{CO}\right] /\left[{ }^{13} \mathrm{CO}\right]=75$ (Wilson \& Rood 1994), 

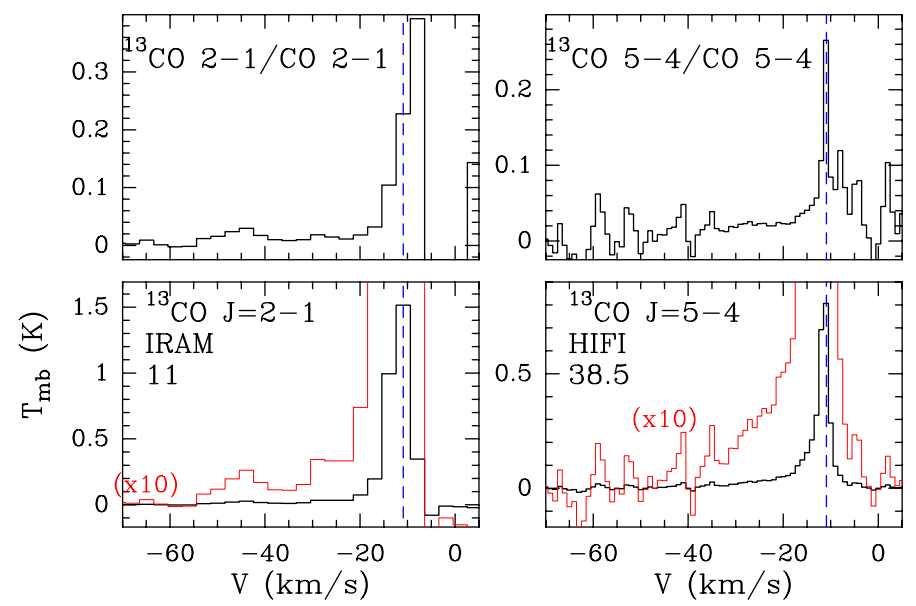

Fig. 4. Bottom: ${ }^{13} \mathrm{CO} J=2-1$ and $5-4$ emission detected towards HH377. The dashed lines (in blue) mark the peak velocity of the cloud emission $\left(v=-10.9 \mathrm{~km} \mathrm{~s}^{-1}\right)$; we have indicated the observatory and the telescope HPBW in arcsec on the top right hand side. Top: intensity line ratio of ${ }^{13} \mathrm{CO} /{ }^{12} \mathrm{CO} J=2-1$ and $5-4$ as a function of velocity, between -70 and $+5 \mathrm{~km} \mathrm{~s}^{-1}$.

these ratios correspond to line opacities of $\tau_{21}^{12}=8$ and 2.1 $\left(\tau_{54}^{12}=3.5\right.$ and 2.2), respectively. Both line ratios decrease sharply with increasing velocity, and vary little for velocities beyond $-20 \mathrm{~km} \mathrm{~s}^{-1}$, with typical values of 0.015 and 0.22 for the $J=2-1$ and 5-4 transitions, respectively. Overall, both transitions can be regarded as optically thin with $\tau_{21}^{12} \leq 1$ and $\tau_{54}^{12}=1.1$ over this velocity range. The ratio appears to decrease again for velocities greater than $-40 \mathrm{~km} \mathrm{~s}^{-1}$, corresponding to lower optical depths. The signal-to-noise ratio of the ${ }^{13} \mathrm{CO}$ data is not sufficient to accurately estimate the line opacity.

Therefore, the line opacity decreases strongly with increasing velocity, a result similar to that obtained by van Kempen et al. (2009a). We conclude that the $J=2-1$ and 5-4 line emissions from the outflow cavity are optically thin, except for velocities close to ambient, between -15 and $-10.9 \mathrm{~km} \mathrm{~s}^{-1}$.

Because of the spatial overlap between the two outflows from Cep E, with rather different excitation conditions, it must be kept in mind that these opacities may not reflect the optical depth of the emission from the high-velocity outflow. This point is addressed in Sect. 4.4.3.

\subsection{Rotational diagram analysis}

In a first step, we estimated the $\mathrm{CO}$ excitation conditions in each outflow component from a simple rotational diagram analysis. The CO line fluxes corrected for the main-beam filling factor are given in Table 2. The rotational diagrams are displayed in Fig. 5. The CO column densities derived in this analysis are therefore values averaged over the source emitting region.

In the jet (top panel of Fig. 5), the flux distribution of the CO lines can be approximately modeled by two components. A component with a rotational temperature $T_{\text {rot }} \simeq 70 \mathrm{~K}$ and column density $N(\mathrm{CO})=9 \times 10^{16} \mathrm{~cm}^{-2}$ accounts for the emission in the range $J_{\text {up }}=1-5$. It fails, however, to account for the emission of the higher-excitation lines $J_{\text {up }} \geq 9$. The emission of this line is well fit by a second gas component of higher excitation with $T_{\text {rot }}=240 \mathrm{~K}$ and of lower column density $N(\mathrm{CO}) \simeq 2 \times 10^{16} \mathrm{~cm}^{-2}$.

In the outflow cavity, a similar pattern with two excitation components is observed (bottom panel of Fig. 5). The flux distribution of the low- $J$ CO lines is well described by a gas
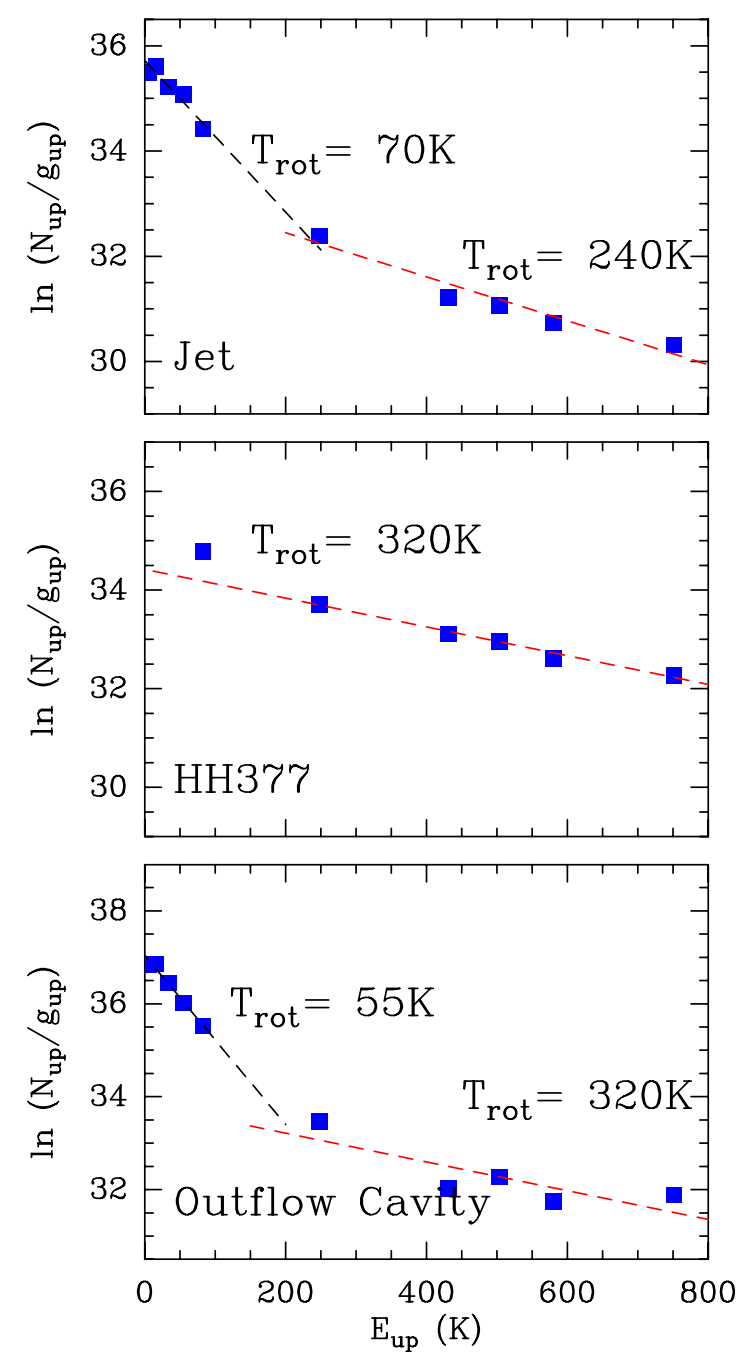

Fig. 5. Rotational diagram of the $\mathrm{CO}$ emission in the three components of the outflow: the jet (top), the terminal shock (middle), and the outflow cavity (bottom).

component with $T_{\text {rot }}=55 \mathrm{~K}$ and $N(\mathrm{CO})=2.5 \times 10^{17} \mathrm{~cm}^{-2}$, whereas the emission of the higher- $J$ lines arises from with gas with $T_{\text {rot }}=200-320 \mathrm{~K}$ and $N(\mathrm{CO}) \simeq(5.6-7.6) \times 10^{16} \mathrm{~cm}^{-2}$. The fit to the high- $J$ lines suffers large uncertainties and we cannot exclude the presence of higher excitation gas components. We speculate that this emission most likely arises from the shocked gas in the cavity walls, as suggested by the good match between the spatial distributions of the $\mathrm{H}_{2}$ pure rotational lines and the CO $J=2-1$ line emission, obtained with Spitzer and the PdBI, respectively.

The two regimes of excitation detected in the jet and the outflow cavity intersect at $E_{\text {up }} \simeq 150-200 \mathrm{~K}$; hence, the millimeter $\left(J_{\text {up }} \leq 3\right)$ and submillimeter $\left(J_{\text {up }} \geq 9\right)$ transitions of CO probe physically different regions both in the jet and in the entrained gas. Therefore, great care must be taken when modeling the emission of the different $\mathrm{CO}$ transitions as it can introduce severe bias in the analysis (Gomez-Ruiz et al. 2012).

Last, in HH377, only one gas component of column density $N(\mathrm{CO}) \simeq 1.3 \times 10^{17} \mathrm{~cm}^{-2}$ and $T_{\text {rot }}=320 \mathrm{~K}$ is detected.

\subsection{Large velocity gradient analysis}

In a second step, we have better constrained the CO column density, the $\mathrm{H}_{2}$ density, and the kinetic temperature in the outflowing 
gas, using the radiative transfer code MADEX in the large velocity gradient (LVG) approximation. We used the $\mathrm{CO}-\mathrm{H}_{2}$ collisional coefficients of Yang et al. (2010) and built a grid of models with density between $10^{4} \mathrm{~cm}^{-3}$ and $10^{7} \mathrm{~cm}^{-3}$ and temperature between $30 \mathrm{~K}$ and $1000 \mathrm{~K}$ to determine the region of minimum $\chi^{2}$ as a function of density and temperature. We adopted a typical linewidth $\Delta v$ of 13 and $15 \mathrm{~km} \mathrm{~s}^{-1}$ for the jet and the shock, respectively, as derived from a simple Gaussian fit to the CO lines. Since the CO line profiles from the outflow cavity are far from a Gaussian line, we analyzed the emission by velocity intervals between -20 and $-17 \mathrm{~km} \mathrm{~s}^{-1}$, and from -50 to -20 by interval of $5 \mathrm{~km} \mathrm{~s}^{-1}$. The physical conditions of the three outflow components are summarized in Table 2.

\subsubsection{The jet}

We first attempted, but failed, to find a reasonable fit of all the CO line fluxes with one single set of parameters $\left(n\left(\mathrm{H}_{2}\right), T_{\text {kin }}\right.$, $N(\mathrm{CO})$ ). In agreement with our rotational diagram analysis (see top panel of Fig. 5) which suggest two regimes of excitation conditions, we modeled the emission of the low- $(J=1-0$ to $5-4)$ and high- $(J=9-8,12-11,13-12,14-13,16-15)$ excitation transitions separately. The results are shown in the top panel of Fig. 6.

In the low-excitation gas, the best-fit solution $\left(\chi^{2}=0.54\right)$ is obtained for $T_{\text {kin }}=100 \mathrm{~K}$ and $n\left(\mathrm{H}_{2}\right)=5 \times 10^{4} \mathrm{~cm}^{-3}$, and $N(\mathrm{CO})=10^{17} \mathrm{~cm}^{-2}$, close to the local thermodynamical equilibrium (LTE) determination (see Sect. 4.2). We find acceptable solutions for gas kinetic temperatures in the range $80-100 \mathrm{~K}$ and $\mathrm{H}_{2}$ jet density $\approx(0.5-1.0) \times 10^{5} \mathrm{~cm}^{-3}$. Solutions with a much higher temperature $\geq 200 \mathrm{~K}$ and lower density $\leq 2 \times 10^{4} \mathrm{~cm}^{-3}$, although possible, are less likely, as indicated by the higher values of $\chi^{2}$, on the order of 0.8 . modeling predicts that all the transitions are optically thin; the highest opacities are found for the $J=5-4$ and $6-5$ transitions with $\tau \simeq 0.4$. This is consistent with our results on the opacity of the $J=2-1$ transition in Sect. 4.1.

A second component at higher temperature is needed in order to account for the emission of the high excitation lines (see Fig. 6). The best-fit solution $\left(\chi^{2}=0.44\right)$ is obtained for a gas column density $N(\mathrm{CO})=1.5 \times 10^{16} \mathrm{~cm}^{-2}$ at $T_{\text {kin }}=650 \mathrm{~K}$ and $n\left(\mathrm{H}_{2}\right)=3 \times 10^{4} \mathrm{~cm}^{-3}$. The physical conditions are less well constrained as solutions with $T_{\text {kin }}$ in the range $400-750 \mathrm{~K}$ and $n\left(\mathrm{H}_{2}\right)$ in the range $(0.5-1.0) \times 10^{6} \mathrm{~cm}^{-3}$ are also possible. All the lines are extremely optically thin $(\tau \ll 1)$. For solutions in the hightemperature regime, the gas density is comparable to the density of the lower-excitation jet component, $\approx 10^{5} \mathrm{~cm}^{-3}$. As discussed below in Sect. 5, we propose that the high-excitation CO emission arises from internal shocks along the jet. Indeed, complementary observations from specific shock tracers in the jet favor solutions in the high-density-low-temperature regime (Lefloch et al., in prep.).

\subsubsection{HH377}

The CO fluxes of the transitions between $J=5-4$ and $J=$ $16-15$ are well reproduced by one gas component of uniform density and temperature. The best-fit solution $\left(\chi^{2}=1.1\right)$ is found for a gas column density $N(\mathrm{CO}) \simeq 10^{17} \mathrm{~cm}^{-2}$ at $T_{\text {kin }}=$ $460 \mathrm{~K}$ and density $n\left(\mathrm{H}_{2}\right) \simeq 10^{6} \mathrm{~cm}^{-3}$. We note that solutions with $T_{\text {kin }}$ temperature as high as $\approx 1000 \mathrm{~K}$, and a density as low as $\approx 2.5 \times 10^{5} \mathrm{~cm}^{-3}$, with $\chi^{2}$ values $\leq 2.5$, can also account for the CO line fluxes. Good solutions $\left(\chi^{2} \leq 1.3\right)$ are found for $T_{\mathrm{kin}}$ in the range $400-500 \mathrm{~K}$ and $n\left(\mathrm{H}_{2}\right)$ in the range $(1-2) \times 10^{6} \mathrm{~cm}^{-3}$.
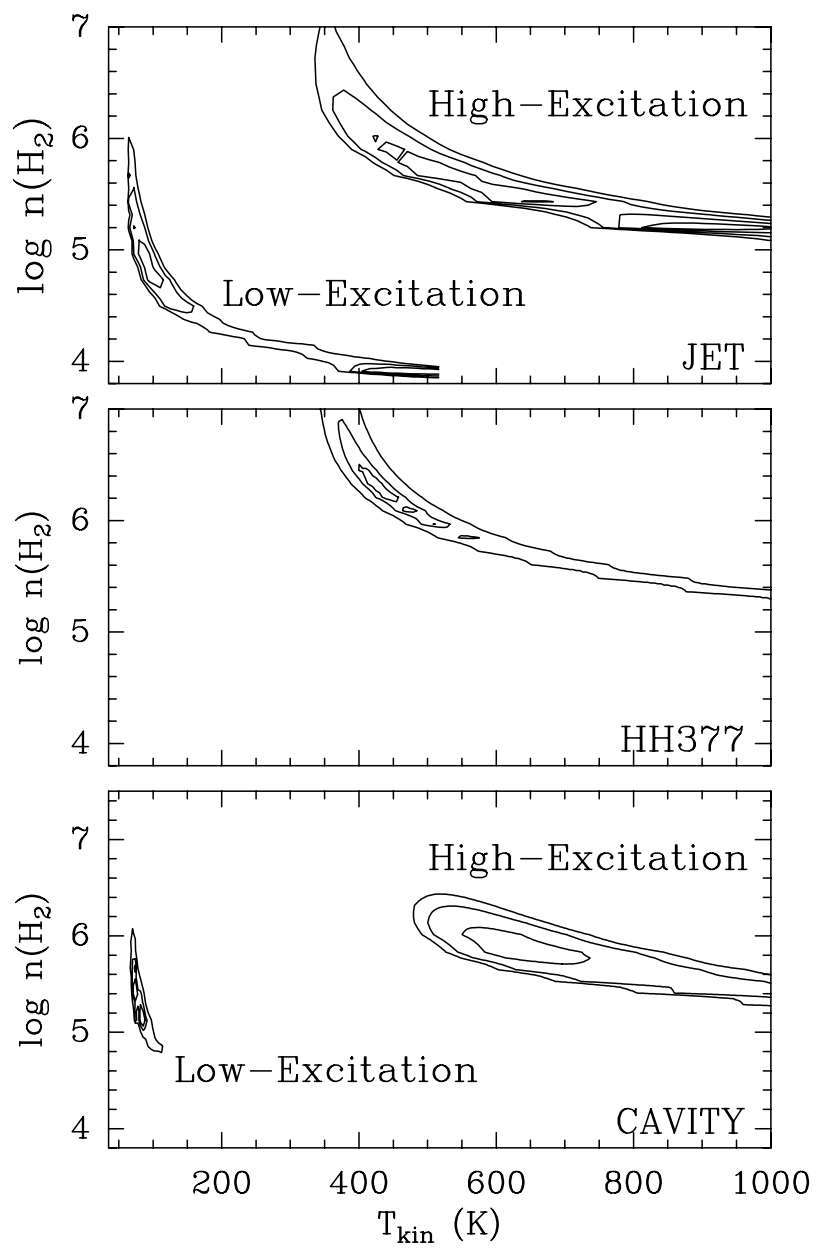

Fig. 6. $\chi^{2}$ distribution of LVG slab models for the three components of the outflow. Top: the jet: contour levels at 0.6, 0.7, 0.8 and at 0.55, 0.65, 0.75 are drawn for the low-excitation component $\left(\chi_{\min }^{2}=0.54\right)$ and the high-excitation component $\left(\chi_{\min }^{2}=0.44\right)$, respectively. Middle: HH377: contour levels are $1.25,1.5$, and $2.5\left(\chi_{\min }^{2}=1.1\right)$. Bottom: the cavity: only emission between -20 and $-17 \mathrm{~km} \mathrm{~s}^{-1}$ is considered. Contour levels at $0.08,0.12,0.15$ and $0.6,1.2,2.4$ are drawn for the high- and lowexcitation components, respectively $\left(\chi_{\min }^{2}=0.08\right.$ and 0.6 , respectively).

All the CO transitions are predicted to be optically thin with $\tau \ll 1$.

Based on observations of the [OI] lines $\lambda 6300,63 \mu \mathrm{m}$ and [SII] line $\lambda \lambda 6717 / 31$ lines, Moro-Martin et al. (2001) concluded that HH377 is associated with a dissociative J-type shock with an estimated pre-shock density of $\sim 10^{5} \mathrm{~cm}^{-3}$ (Ayala et al. 2000). Dissociative shock models (Hollenbach \& McKee 1989; Gusdorf et al. 2015) predict the CO emission to arise from the molecular reformation zone in the post-shocked gas, with temperatures in the range $400-500 \mathrm{~K}$. Hence, both previous work and shock models favor solutions with a low temperature $(\simeq 400-500 \mathrm{~K})$ and high density $\left((1-2) \times 10^{6} \mathrm{~cm}^{-3}\right)$.

\subsubsection{The cavity}

We first considered the flux of the transitions $J=1-0,2-1,3-2$, and $9-8$ between -20 and $-17 \mathrm{~km} \mathrm{~s}^{-1}$. These transitions were observed towards the same position and at a common angular resolution of $\approx 20^{\prime \prime}$. We ran our LVG code with a $\chi^{2}$ minimization procedure varying the main-beam filling factor, $\mathrm{CO}$ column 
Table 4. Summary of the physical conditions in the CepE outflow components: the jet, the terminal shock HH377 and the entrained gas (the outflow cavity).

\begin{tabular}{llrrr}
\hline \hline & & Jet & HH377 & Outflow cavity \\
\hline Global & Size $\left(^{\prime \prime}\right)$ & $1.7 \times 21$ & 4.5 & $22 \times 10$ \\
parameters & Velocity range $\left(\mathrm{km} \mathrm{s}^{-1}\right)$ & $-140 ;-100$ & $-90 ;-50$ & $-100 ;-11$ \\
& Mass $\left(M_{\odot}\right)$ & 0.02 & 0.006 & 0.32 \\
& Radial momentum $\left(M_{\odot} \mathrm{km} \mathrm{s}^{-1}\right)$ & 1.7 & 0.4 & 2.6 \\
Low-excitation & $\mathrm{T}_{\text {kin }}(\mathrm{K})$ & & & $55-85$ \\
component & $N(\mathrm{CO})\left(\mathrm{cm}^{-2}\right)$ & $80-100$ & - & $7(17)$ \\
& $n\left(\mathrm{H}_{2}\right)\left(\mathrm{cm}^{-3}\right)$ & $9(16)$ & - & $(1-8)(5)$ \\
High-excitation & $\mathrm{T}_{\text {kin }}(\mathrm{K})$ & $(0.5-1.0)(5)$ & - & $500-1500$ \\
component & $N(\mathrm{CO})\left(\mathrm{cm}^{-2}\right)$ & $400-750$ & $400-500$ & $6(16)$ \\
& $n\left(\mathrm{H}_{2}\right)\left(\mathrm{cm}^{-3}\right)$ & $1.5(16)$ & $1.0(17)$ & $(1-5)(6)$ \\
\hline
\end{tabular}

Notes. Two physical components, of low- and high-excitation excitation respectively, are detected in both the jet and the outflow cavity. Only one component is detected towards HH377 (see text). We adopt the convention $a(b)=a \times 10^{b}$.

density, temperature, and density; the minimum $\chi^{2}$ is obtained for a filling factor $0.17-0.20$. This value is very close to the determination obtained independently by means of the interferometric map of the Plateau de Bure, as explained in Sect. 4.1 (Table 2); we are confident in the method adopted.

Because the intensity distribution between -50 and $-14 \mathrm{~km} \mathrm{~s}^{-1}$ is far from a Gaussian distribution, we decided to analyze the $\mathrm{CO}$ line intensities integrated over narrower velocity intervals: between -14 and $-17,-17$ and $-20,-20$ and $-25,-25$ and $-30,-30$ and $-35,-35$ and -40 , and -50 and $-40 \mathrm{~km} \mathrm{~s}^{-1}$. Again, we found that it is possible to account for the emission of all the transitions from $J=1-0$ up to $J=9-8$ with one single component. The best fitting solution is rather good in the low velocity range $\left(\chi^{2} \simeq 0.45\right)$. The best fitting solution cannot account for the intensity of the high-excitation lines $J=12-11$ to $16-15$. We note that the $J=9-8$ flux lies at the intersection of the two regimes of excitation found in the rotational diagram (see Fig. 5). Therefore, we have searched for the best fitting solutions which allow each excitation regime to be reproduced separately, $J=1-0$ to $5-4$, and $J=12-1$ to $16-15$, respectively, with the additional constraints that a) the resulting flux for the $J=9-8$ was consistent with the observational determination, and b) the opacity of the CO $J=5-4$ agrees with the observations. The physical parameters for each velocity interval are summarized in Table 3 and the best fitting solutions for each velocity interval are displayed in Figs. B.1-B.2. We show in Fig. B3 the best fitting solutions for both components and the total fit to the full spectral line flux distribution for each velocity interval.

The emission of the low-excitation gas is well described by a gas layer with a kinetic temperature in the range $55 \mathrm{~K}$ to $85 \mathrm{~K}$ and density of $(1-8) \times 10^{5} \mathrm{~cm}^{-3}$ (Fig. B.1). The $\mathrm{CO}$ column density decreases with velocity from $10^{17} \mathrm{~cm}^{-2}$ between -20 and $-17 \mathrm{~km} \mathrm{~s}^{-1}$ down to $3 \times 10^{16} \mathrm{~cm}^{-2}$ between -50 and $-40 \mathrm{~km} \mathrm{~s}^{-1}$. The $\mathrm{H}_{2}$ density is reasonably well constrained in the low-velocity range up to $-30 \mathrm{~km} \mathrm{~s}^{-1}$, with $n\left(\mathrm{H}_{2}\right) \simeq$ $(1-4) \times 10^{5} \mathrm{~cm}^{-3}$. If we extrapolate the excitation conditions derived between -14 and $-17 \mathrm{~km}$ to the lower velocity gas between -14 and $-11 \mathrm{~km} \mathrm{~s}^{-1}$, we estimate a typical column density $2 \times 10^{17} \mathrm{~cm}^{-2}$. The total gas column density is then $N(\mathrm{CO}) \simeq 7 \times 10^{17} \mathrm{~cm}^{-2}$.

We give in Table 3 the opacities of the $J=2-1$ and 5-4 transitions, as predicted by our simple modeling. The agreement with the observational determination is found satisfying for the
$J=5-4$ transition, with $\tau_{54}^{12}$ on the order of 1 between -20 and $-30 \mathrm{~km} \mathrm{~s}^{-1}$. On the contrary, our modeling predicts the $J=2-1$ transition to be optically thin even in the low-velocity range, whereas observations indicate $\tau_{21}^{12}=0.8-8$ between -14 and $-20 \mathrm{~km} \mathrm{~s}^{-1}$. At higher velocities, the agreement is reasonable. As mentioned in Sect. 4.2, the emission of the lowexcitation lines is contaminated by the contribution of the largescale, lower-excitation outflow. The physical conditions of this outflow were derived by Ladd \& Hodapp (1997) from CO $J=$ $1-0$ and $2-1$ observations, who reported a typical temperature $T_{\text {kin }} \simeq 20 \mathrm{~K}$ and column density $N(\mathrm{CO}) \simeq 4 \times 10^{15} \mathrm{~cm}^{-2}$. Assuming a typical outflow density of a few $10^{4} \mathrm{~cm}^{-3}$, LVG calculations of the $\mathrm{CO}$ emission shows that the gas emits mainly in the lines $J_{\text {up }}=1$ to 4 . As a result, in the velocity range -11 to $-20 \mathrm{~km} \mathrm{~s}^{-1}$, the emission of both the $\mathrm{CO}$ and ${ }^{13} \mathrm{CO}$ lines could be biased by the lower-excitation emission from the large-scale outflow, whereas the higher-excitation lines, $J=5-4$ and higher, would not be affected as they arise mainly from the compact, high-excitation outflow. In all cases, the modeling presented here is very crude; an approach taking into account the variations of temperature and density as a function of location inside the outflow cavity would be far more realistic. Such modeling would be at the cost of many additional parameters, which cannot be constrained by the observations discussed here owing to their limited angular resolution.

The emission of high-excitation lines is accounted for by gas at temperatures ranging from $\simeq 500 \mathrm{~K}$ up to $1500 \mathrm{~K}$, and density in the range $(1-5) \times 10^{6} \mathrm{~cm}^{-3}$ (see Fig. 6; also Fig. B.2). The CO column density decreases with velocity from $6.5 \times 10^{15} \mathrm{~cm}^{-2}$ between -20 and $-17 \mathrm{~km} \mathrm{~s}^{-1}$ down to $8.5 \times 10^{15} \mathrm{~cm}^{-2}$ between -50 and $-40 \mathrm{~km} \mathrm{~s}^{-1}$. Proceeding as above and extrapolating the excitation conditions derived between -14 and $-17 \mathrm{~km}$ to the lower velocity gas between -14 and $-11 \mathrm{~km} \mathrm{~s}^{-1}$, we obtain a total column density $6 \times 10^{16} \mathrm{~cm}^{-2}$. The emission of this component is predicted to be highly optically thin in all the $\mathrm{CO}$ transitions: $\tau \ll 1$. Our $\chi^{2}$ minimization analysis suggests a gradient of excitation in the outflowing gas as higher-temperature solutions are favored when the velocity range increases (see Table 4 and Fig. B.2). Complementary observations from high-density gas tracers will be useful to better constrain the density and the temperature distributions in the entrained gas. The best fitting temperature and $\mathrm{CO}$ column density are in good agreement with the results of Moro-Martin et al. (2001), based on ISO/LWS observations of the CO transitions $J=14-13$ up to $J=33-32$ at 
$80^{\prime \prime}$ resolution towards the southern lobe of CepE. These authors found $T_{\text {kin }}=1200 \mathrm{~K}$ and $N(\mathrm{CO})=10^{17} \mathrm{~cm}^{-2}$ from a large velocity gradient analysis. These results are also consistent with the analysis of the pure $\mathrm{H}_{2}$ rotational lines by the same authors.

\subsection{Mass and momentum budget}

We have estimated the mass contained in the different outflow components as follows.

For the jet, we integrated the $\mathrm{CO} J=2-1$ emission in the southern jet lobe, as mapped with the PdBI, using the following relation under the hypothesis of LTE:

$N(\mathrm{CO})=1.06 \times 10^{13} T_{\text {ex }} \exp \left(16.5 / T_{\text {ex }}\right) \int T_{B} \mathrm{~d} v \mathrm{~cm}^{-2}$

(see Bachiller et al. 1990). This relation is valid only in the optically thin regime and can be applied safely to the jet, as its emission is optically thin (see Sect. 4.1). Adopting $T_{\mathrm{ex}}=70 \mathrm{~K}$ (see Sect. 4.3), we obtain $0.02 M_{\odot}$ for the jet mass. Given the dynamical age estimated in Sect. 3.2, the mass-loss rate associated with the jet is then $\dot{M}=2 \times 10^{-5} M_{\odot} \mathrm{yr}^{-1}$.

For the outflow cavity, the interval of integration was decomposed into two velocity intervals:

- Between -100 and $-50 \mathrm{~km} \mathrm{~s}^{-1}$, we applied the above relation to the CO $J=2-1$ emission which we corrected for the line opacity $\tau^{12}$. To do so, we modeled the variations of $\tau^{12}$ as a function of velocity by a simple synthetic profile and introduced the correcting factor $\tau^{12} /\left(1-\exp \left(-\tau^{12}\right)\right)$ in the previous relation. We find a mass of $0.02 M_{\odot}$.

- Between -50 and $-11 \mathrm{~km} \mathrm{~s}^{-1}$, we adopted the total CO column density obtained in our LVG analysis (Sect. 4.4) and adopted the size of the outflow cavity, as estimated with the PdBI. The mass of the outflowing gas between -50 and -20 is $\approx 0.30 M_{\odot}$.

Hence, we estimate a total mass of $\simeq 0.32 M_{\odot}$ for the outflow cavity gas. Our estimate of the outflow cavity mass is somewhat larger by a factor 6-10 to the previous determinations by Moro-Martin et al. (2001) and Ladd \& Hodapp (1997), who found $0.05 M_{\odot}$ and $0.03 M_{\odot}$, respectively. Such discrepancies can easily be reconciled. These authors adopted a CO excitation temperature of $20 \mathrm{~K}$, much lower than the $55 \mathrm{~K}$ estimated in the present work, and neglected any correction for line opacity effects. The discrepancy with the estimate of Moro-Martin et al. (2001) is then $\approx 2$, which is satisfying taking into account that their estimate is based on ${ }^{13} \mathrm{CO}$ interferometric observations of the outflow, uncorrected for flux filtering and primary beam attenuation.

We proceeded in the same way in order to estimate the radial momentum associated with the jet and the outflow cavity. In the jet, we found $1.7 M_{\odot} \mathrm{km} \mathrm{s}^{-1}$. This is very similar to a crude estimate based on the mass of the jet and the mean jet velocity with respect to the ambient cloud. In the outflow cavity, the total radial momentum carried away amounts to $\approx 2.6 M_{\odot} \mathrm{km} \mathrm{s}^{-1}$, a value very similar to that in the jet. This very good agreement suggests that the jet momentum is transferred to the entrained gas in the low-velocity outflow with a high efficiency. It supports the idea that the Cep E outflow is driven by the molecular jet through the bowshock HH377, in agreement with the model of Raga \& Cabrit (1993).

For the terminal bowshock HH377, the mass and momentum estimates are more uncertain as the $J=2-1$ line appears to be dominated by the entrained gas. Hence, we obtained a rough estimate of the mass from the source-averaged CO column density derived from our LVG analysis and the typical size of $5^{\prime \prime}$ estimated from our interferometric data. We found $0.006 M_{\odot}$. An estimate of the momentum of $0.4 M_{\odot} \mathrm{km} \mathrm{s}^{-1}$ was obtained by adopting a mean value of $60 \mathrm{~km} \mathrm{~s}^{-1}$ with respect to the ambient cloud (see also Fig. 3).

\section{The jet structure}

In the jet, the high- $J$ CO transitions $\left(J_{\text {up }} \geq 9\right)$ trace mainly the warm gas component (Fig. 5). Our PdBI map of the CO 2-1 emission reveals the presence of bright emission knots along the jet (Figs. 1 and 2). The main-beam solid angle of the high- $J$ CO lines $(J \geq 9)$ encompasses two such knots, peaking at $\delta=-16^{\prime \prime}$ and $\delta=-20^{\prime \prime}$, respectively, which present a typical size of $2^{\prime \prime}-4$ " (see Figs. 1,2). Variations of the centroid of emission and of the line profiles are also observed, on the order of $10 \mathrm{~km} \mathrm{~s}^{-1}$, showing evidence of the presence of internal motions and a complex small-scale structure inside the jet. Interestingly, the presence of warm $\left(T_{\text {kin }} \simeq 200 \mathrm{~K}\right)$, dense $\left(\simeq 10^{6} \mathrm{~cm}^{-3}\right)$ clumps of gas of arcsec size, associated with $\mathrm{H}_{2} \mathrm{O}$ and $\mathrm{SiO}$ emission was reported by Lefloch et al. (2011) along the protostellar jet. These previous results suggest that the emission of the high- $J$ CO lines could arise from shocked clumps of gas along the jet.

We tested this hypothesis by comparing the CO flux spectral distribution of the hot jet component with the predictions of a small grid of shock models, following a method extensively presented in e.g. Gusdorf et al. (2011, 2012). In this simplified approach, clumps of shocked gas propagating along the jet are compared to one-dimensional shock models. This approach is justified by the lack of information on the internal structure of the jet. The fluxes of the low- $J$ transitions were estimated from our LTE modeling (Sect. 4.2). The grid consists of steady-state J-type and C-type models from Gusdorf et al. (2015) and also from a set of non-stationary models (Lesaffre et al. 2004) with input parameters adapted to the present region: pre-shock nuclei density $n_{\mathrm{H}}=10^{4}, 5 \times 10^{4}, 10^{5}, 5 \times 10^{5} \mathrm{~cm}^{-3}$; magnetic field (transverse to the shock direction) given by $B=\left(\frac{n_{\mathrm{H}}}{1 \mathrm{~cm}^{-3}}\right)^{1 / 2} \mu \mathrm{G}$; and shock velocity $v_{\mathrm{s}}=20,25,30 \mathrm{~km} \mathrm{~s}^{-1}$, well in the range of internal motions detected in the PdBI data. The non-stationary shock models present combined characteristics of both stationary C- and J-type models, and are consequently called "CJ-type" shock models. For these models, the age is an additional input parameter; we chose to explore an age less than 1000 years, the dynamical age of HH 377.

The observational $\mathrm{CO}$ spectral flux distribution reaches its maximum at $J_{\text {up }}=9$. There are no J-type shock models that can account for the $\mathrm{CO}$ flux distribution: the corresponding shock layers are too thin to generate sufficient $\mathrm{CO}$ emission to match the data. We searched for a CJ-type solution to fit the CO line fluxes given in Table 2, i.e. we assumed that the high-excitation component has the same filling factor as the low-excitation gas, such as that mapped with the PdBI map (relative filling factor of 1). The model grid is displayed in Fig. 7 and the observational fluxes are drawn with filled squares. We find that CJ-type shocks propagating into a pre-shock gas of density $n_{\mathrm{H}}=(0.5-1.0) \times$ $10^{5} \mathrm{~cm}^{-3}$, corresponding to $n\left(\mathrm{H}_{2}\right)=(2.5-5) \times 10^{4} \mathrm{~cm}^{-3}$ and $B=(0.2-0.3) \mathrm{mG}$, with a velocity $v_{\mathrm{s}} \simeq 20-30 \mathrm{~km} \mathrm{~s}^{-1}$ match rather well the observed $\mathrm{CO}$ fluxes (see bottom left and upper right panel in Fig. 7, and top panel in Fig. 8). Such a pre-shock density is consistent with our determination of the density in the bulk material of the jet (Table 2). The pre-shock density is 

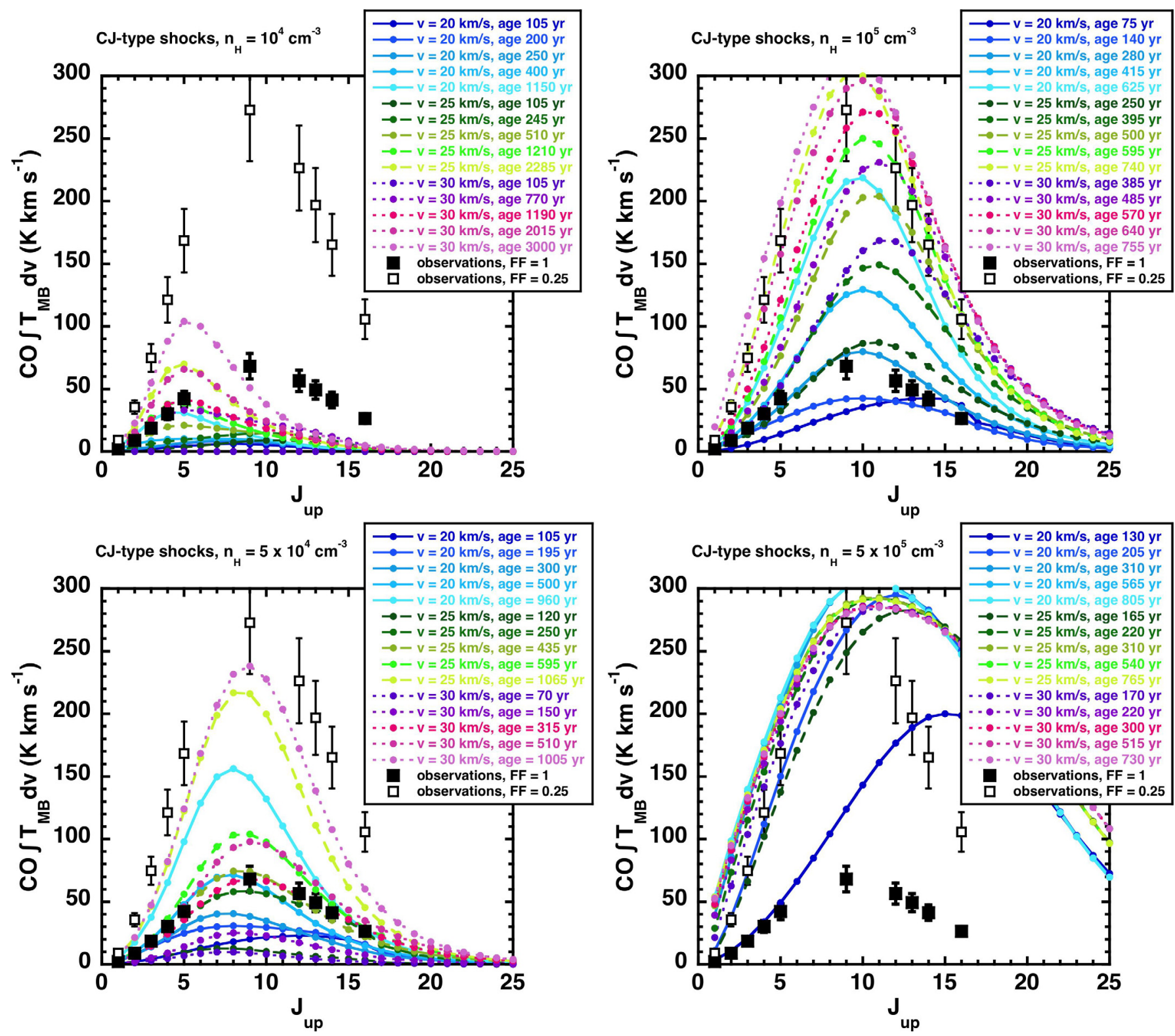

Fig. 7. CO velocity-integrated intensity diagrams predicted by non-stationary CJ-type shock models for various pre-shock densities: $n_{\mathrm{H}}=10^{4}$ (upper left panel), $5 \times 10^{4} \mathrm{~cm}^{-3}$ (lower left panel), $10^{5}$ (upper right panel), $5 \times 10^{5} \mathrm{~cm}^{-3}$ (lower right panel), and $B=\sqrt{n_{\mathrm{H}}\left(\mathrm{cm}^{-3}\right)} \mu \mathrm{G}$. The observational data are drawn with filled (empty) squares for a filling factor of 1 (of 0.25 ). The shock velocity and age are given in each panel, as is the color code.

rather well constrained: for pre-shock gas densities lower than $n_{\mathrm{H}}=10^{4} \mathrm{~cm}^{-3}$ (upper left panel in Fig. 7), CJ-type shocks fail to produce bright enough emission in the high- $J$ CO lines. On the contrary, higher pre-shock gas densities $n_{\mathrm{H}}=5 \times 10^{5} \mathrm{~cm}^{-3}$ (bottom right panel in Fig. 7) tend to produce $\mathrm{CO}$ lines much brighter than those observed, by a factor of about 5 . For a filling factor of 1 , the best agreement is obtained for CJ-type shocks at a relatively early age of $220-450 \mathrm{yr}$, as is shown in the upper panel in Fig. 8. Our models predict that the density of the $\mathrm{CO}$ emitting layer lies in the range from a few $10^{5}$ to $10^{6} \mathrm{~cm}^{-3}$, which is consistent with the physical conditions derived from our LVG analysis (Sect. 4.3).

In what precedes, we assumed that the shocked gas has the same filling factor as the bulk material of the CO jet. The size of the shock is not well constrained and it could be smaller than the size of the $70 \mathrm{~K}$ component, which dominates the $J=2-1$ jet emission. In this case, the $\mathrm{CO}$ line emission from the shock would be brighter than estimated above (Table 2). In Fig. 7 we have superposed the $\mathrm{CO}$ line fluxes to our model predictions under the assumption that the relative filling factor (FF) is 0.25 (empty square symbol) and have compared these data points to our models. A filling factor of 0.25 corresponds to a typical size $4^{\prime \prime}-5^{\prime \prime}$ for one component, or $\simeq 2^{\prime \prime}$ for two components of similar excitation. Such sizes are compatible with the size of the CO knots in the jet, such as those mapped with the PdBI. We have also chosen to display the best fitting results for this value of the filling factor in the lower panel of Fig. 8. This figure shows that slightly older shock models are then favored (age 595-740 yr), with a pre-shock density more tightly constrained to $n_{\mathrm{H}}=10^{5} \mathrm{~cm}^{-3}$.

Complementary or additional CO line observations ( $J_{\text {up }}=6$, $7,11,12$ are possible from the ground or from SOFIA) would help to more tightly constrain our shock models. Interferometric observations at subarcsec scale of shock tracers such as $\mathrm{SiO}$ 
B. Lefloch et al.: CO emission in the Cep E outflow: the outflow cavity, the bowshock, and the jet

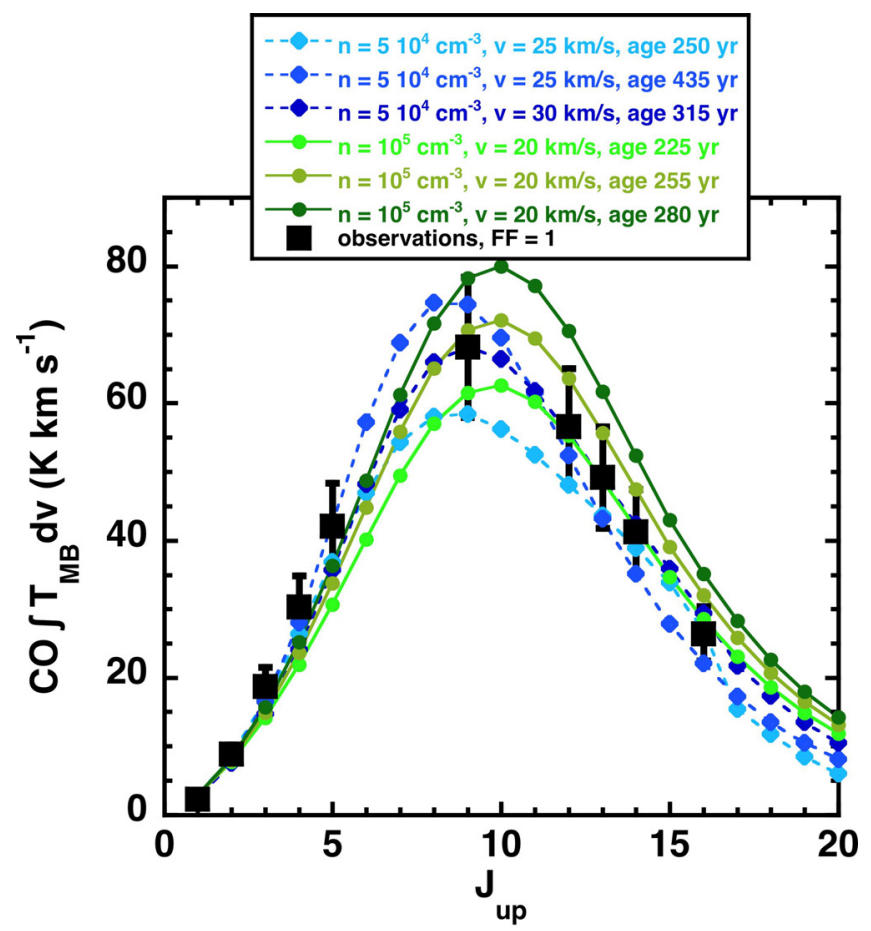

\section{Conclusions}

We have carried out an unprecedented multi-transition study at high spectral resolution of the $\mathrm{CO}$ emission in the southern outflow lobe of the intermediate-mass protostar Cep E-mm, based on observations of transitions in the range $J=1-16$. PdBI observations at arcsec scale of the $J=2-1$ line show that the "highvelocity molecular bullets" reported in a previous work are actually tracing a protostellar jet and the terminal bowshock HH377 where the jet impacts the molecular cloud. The dynamical age of the jet is short, $\approx 960 \mathrm{yr}$, suggesting that the jet is still at an early evolutionary stage.

We have constrained the physical conditions in three outflow components from an LTE and LVG analysis:

- In the jet, of size $21^{\prime \prime} \times 1.7^{\prime \prime}$, the low- $J$ CO emission is dominated by a gas layer of column density $N(\mathrm{CO})=$ $9.0 \times 10^{16} \mathrm{~cm}^{-2}$, density $n\left(\mathrm{H}_{2}\right)=(0.5-1) \times 10^{5} \mathrm{~cm}^{-3}$ and temperature $T_{\text {kin }}=80-100 \mathrm{~K}$; the high- $J$ CO emission is well accounted for by a gas layer of lower column density $N(\mathrm{CO})=1.5 \times 10^{16} \mathrm{~cm}^{-2}$, higher density $\left(n\left(\mathrm{H}_{2}\right)=(0.5-1.0) \times 10^{6} \mathrm{~cm}^{-3}\right)$ and higher temperature $\left(T_{\text {kin }}=400-750 \mathrm{~K}\right)$.

- In the terminal bowshock HH377, of size 4.5", the CO emission is well described by one single gas component of dense gas $\left(n\left(\mathrm{H}_{2}\right)=(1-2) \times 10^{6} \mathrm{~cm}^{-3}\right)$ with temperature in the range $400-500 \mathrm{~K}$ and column density $N(\mathrm{CO})=10^{17} \mathrm{~cm}^{-2}$.

- In the outflow cavity, of size $22^{\prime \prime} \times 10^{\prime \prime}$, the low- $J$ CO emission is dominated by a gas layer of column density $N(\mathrm{CO}) \simeq$ $7 \times 10^{17} \mathrm{~cm}^{-2}$, density $n\left(\mathrm{H}_{2}\right) \simeq(1-8) \times 10^{5} \mathrm{~cm}^{-3}$, and temperature $T_{\text {kin }} \simeq 50-85 \mathrm{~K}$; the high- $J \mathrm{CO}$ line emission is accounted for by a gas layer of lower column density $N(\mathrm{CO})=$ $6 \times 10^{16} \mathrm{~cm}^{-2}$, higher density $n\left(\mathrm{H}_{2}\right)=(1-5) \times 10^{6} \mathrm{~cm}^{-3}$, and higher temperature $T_{\text {kin }}=500-1500 \mathrm{~K}$. Evidence of a gradient of excitation in the high-excitation gas suggests a complex thermal structure in the outflow cavity.

Overall, the jet and the outflow cavity appear to carry away the same amount of angular momentum, which supports Cep E as a convincing case of jet bowshock driven outflow.

The presence of hot $\mathrm{CO}$ gas inside the jet can be consistently explained by the presence of internal CJ-shocks propagating at a velocity of $\sim 20-30 \mathrm{~km} \mathrm{~s}^{-1}$ along the jet. This implies that the jet is magnetized with typical magnetic field intensity (transverse to the shock direction) on the order of 200-300 $\mu \mathrm{G}$. Interferometric observations at subarcsec scale of specific shock tracers with NOEMA would help constrain the structure of the protostellar jet and the bowshock HH377.

Acknowledgements. We thank the referee Dr. Tim van Kempen and the Editor Malcolm Walmsley for detailed comments which have helped to improve the manuscript. We also thank the SOFIA operations and the GREAT instrument teams, whose support has been essential for the GREAT accomplishments, and the DSI telescope engineering team. Based on observations made with the NASA/DLR Stratospheric Observatory for Infrared Astronomy, with the IRAM $30 \mathrm{~m}$ telescope and the IRAM Plateau de Bure Interferometer. SOFIA Science Mission Operations are conducted jointly by the Universities Space Research Association, Inc., under NASA contract NAS2-97001, and the Deutsches SOFIA Institut, under DLR contract 50 OK 0901. IRAM is supported by INSU/CNRS (France), MPG (Germany) and IGN (Spain). The James Clerk Maxwell Telescope is operated by the Joint Astronomy Centre on behalf of the Science and Technology Facilities Council of the United Kingdom, the Netherlands Organisation for Scientific Research, and the National Research Council of Canada. This work was partly supported by the CNRS program "Physique et Chimie du Milieu Interstellaire", by the PRIN INAF 2012 JEDI and by the Italian Ministero dell'Istruzione, Università e Ricerca through the grant Progetti Premiali 2012 - iALMA A.I. Gómez-Ruiz is supported by Consejo Nacional de Ciencia y Tecnología, through the program Cátedras CONACYT para Jóvenes Investigadores.

(see e.g. Lefloch et al. 1996, 2011) would help to constrain the size of these shocks and to provide a precise structure of the protostellar jet. 


\section{References}

Ayala, S., Noriega-Crespo, A., Garnavich, P. M., et al., 2000, AJ, 120, 909

Bachiller, R., 1996, ARA\&A, 34, 111

Busquet, G., Lefloch, B., Benedettini, M., et al., 2014, A\&A, 561, A120

Ceccarelli, C., Bacmann, A., Boogert, A., et al., 2010, A\&A, 521, L22

Chini, R., Ward-Thompson, D., Kirk, J. M., et al., 2001, A\&A, 369, 155

Eislöffel, J., Smith, M. D., \& Davis, C. J., 1996, AJ, 112, 2086

Giannini, T., Nisini B., \& Lorenzetti D., 2001, ApJ, 555, 40

Froebrich, D., Smith, M. D., Hodapp, K.-W., \& Eislöffel, J., 2003, MNRAS, 346, 163

Gomez-Ruiz, A. I., Gusdorf, A., Leurini, S., et al., 2012, A\&A, 542, L9

Gomez-Ruiz, A. I., Wyrowski, F., Gusdorf, A., et al., 2013, A\&A, 555, A8

Guan, X., Stutzki, J., Graf, U., et al., 2012, A\&A, 542, L4

Gusdorf, A., Giannini, T., Flower, D. R., et al., 2011, A\&A, 532, A53

Gusdorf, A., Anderl, S., Güsten, R., et al., 2012, A\&A, 542, L19

Gusdorf, A., Riquelme, D., Anderl, S., et al., 2015, A\&A, 575, A98

Hatchell, J., Fuller, G. A., \& Ladd, E. F., 1999a, A\&A, 344, 687

Hatchell, J., Fuller, G. A., \& Ladd, E. F., 1999b, A\&A, 346, 278

Heyminck, S., Graf, U., Güsten, R., et al., 2012, A\&A, 542, L1

Hollenbach, D., \& McKee, C. F., 1989, ApJ, 342, 306

Klein, B., Hochgürtel, S., Krämer, I., et al., 2012, A\&A, 542, L3
Ladd, E. F., \& Hodapp, K.-W., 1997, ApJ, 475, 749

Lefloch, B., Eislöffel, J., \& Lazareff B., 1996, A\&A, 313, L17

Lefloch, B., Cernicharo, J., Pacheco, S., \& Ceccarelli, C., 2011, A\&A, 527, L3

Lefloch, B., Cabrit, S., Busquet, G., et al., 2012, ApJ, 757, L25

Lesaffre, P., Pineau des Forets, G., Godard, B., et al., 2004, A\&A, 427, 147

Moro-Martín, A., Noriega-Crespo, A., Molinari, S., et al., 2001, ApJ, 555, 146

Noriega-Crespo, A., Garnavich, P. M., \& Molinari, S., 1998, AJ, 116, 1388

Noriega-Crespo, A., Moro-martin, A., Carey, S., et al. 2004, ApJS, 154, 402

Noriega-Crespo, A., Raga, A. C., Moro-Martin, A., et al., 2014, New J. Phys., 16, 1005008

Raga, A. C., \& Cabrit, S., 1993, A\&A, 278, 267

Sargent, A., 1977, ApJ, 218, 736

Smith, M. D., Froebrich, D., \& Eislöffel, J., 2003, ApJ, 592, 245

Stark, R., Sandell, G., Beck, Sara C., et al., 2004, ApJ, 608, 341

van Dishoeck, E. F., Kristensen, L. E., Benz, A. O., et al., 2011, PASP, 123, 138

van Kempen, T. A., Hogerheijde, M. R., van Dishoeck, E. F., et al., 2006, A\&A, 454, L75

van Kempen, T., E. F. van Dishoeck, R. Güsten, et al., 2009a, A\&A, 501, 633 van Kempen, T., E. F. van Dishoeck, R. Güsten, et al., 2009b, A\&A, 507, 1442

Visser, R., Kristensen, L. E., Bruderer, S., et al., 2012, A\&A, 537, A55

Wilson, T. L., \& Rood, R. T., 1994, ARA\&A, 32, 191

Yang, B., Stancil, P. C., Balakrishnan, N., \& Forrey R. C., 2010, ApJ, 718, 1062

Yildiz, U. A., Kristensen, L. E., van Dishoeck, E. F., et al., 2013, A\&A, 556, A89

Pages 15 to 21 are available in the electronic edition of the journal at http://www . aanda. org 
B. Lefloch et al.: CO emission in the Cep E outflow: the outflow cavity, the bowshock, and the jet

\section{Appendix A: Complementary single-dish CO observations}

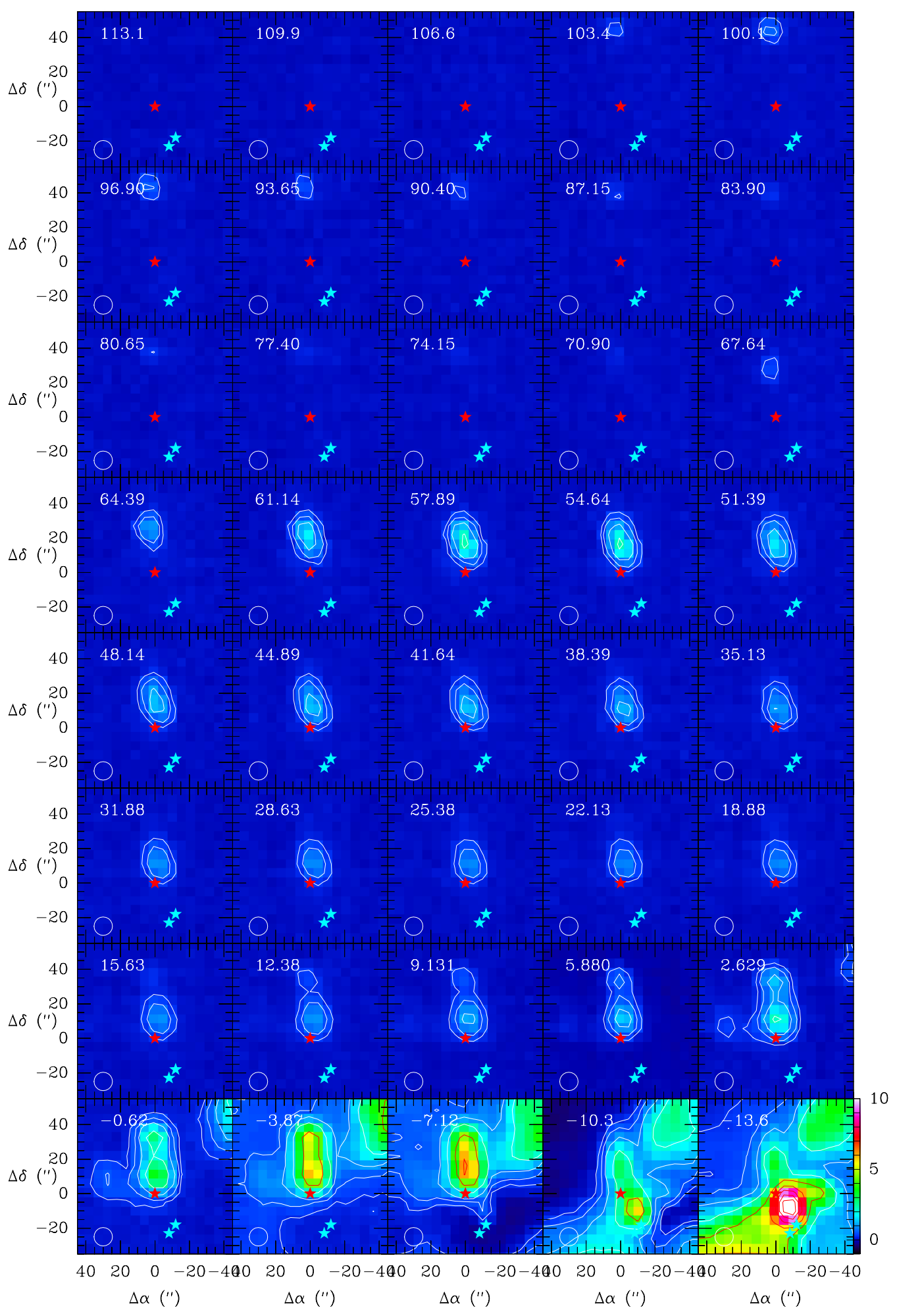

Fig. A.1. Map of the CO $J=2-1$ emission integrated in channels of $3.25 \mathrm{~km}$ from -13.5 to $+113.1 \mathrm{~km} \mathrm{~s}^{-1}$, as observed with the IRAM $30 \mathrm{~m}$ telescope. The position of the protostar is marked by a red star. The positions targeted by GREAT/SOFIA and HIFI/Herschel are marked by blue stars. The size of the telescope HPBW (10.7") is indicated by the white circle. 
A\&A 581, A4 (2015)

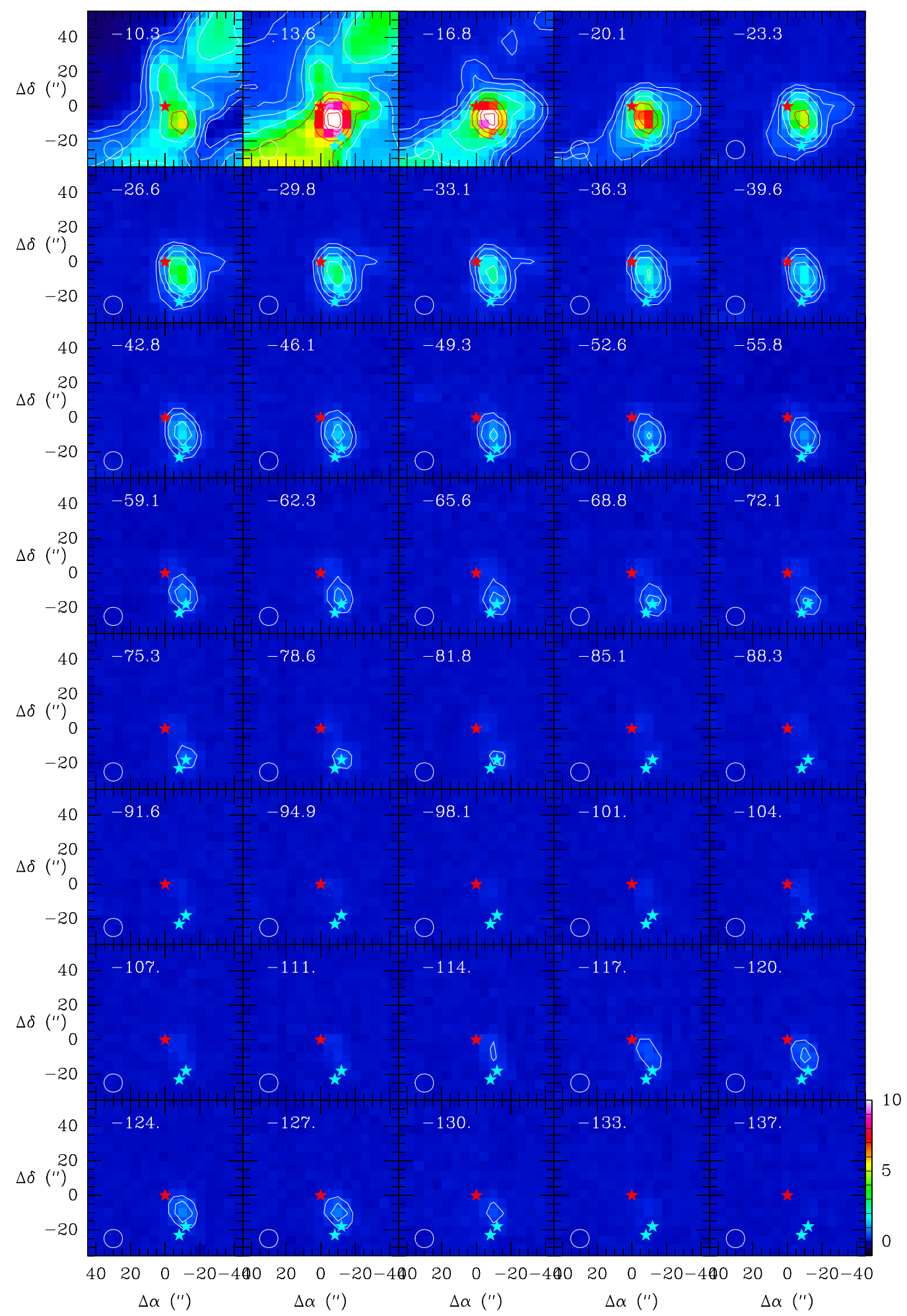

Fig. A.2. Map of the CO $J=2-1$ emission integrated in channels of $3.25 \mathrm{~km}$ from -137.0 to $-10.3 \mathrm{~km} \mathrm{~s}^{-1}$, as observed with the IRAM $30 \mathrm{~m}$ telescope. The position of the protostar is marked by a red star. The positions targeted by GREAT/SOFIA and HIFI/Herschel are marked by blue stars. The size of the telescope HPBW (10.7") is indicated by the white circle. 
B. Lefloch et al.: CO emission in the Cep E outflow: the outflow cavity, the bowshock, and the jet

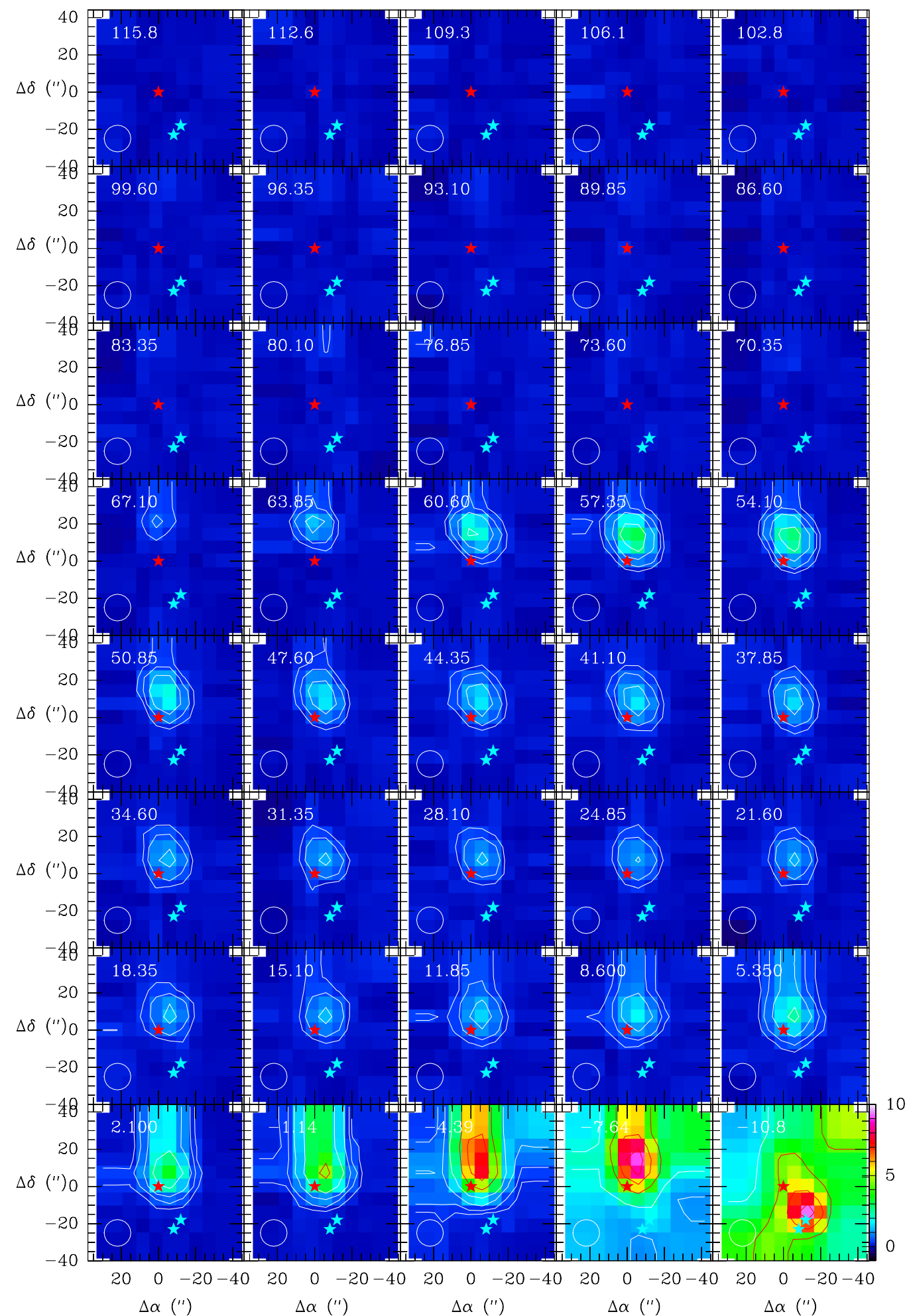

Fig. A.3. Map of the CO $J=3-2$ emission integrated in channels of $3.25 \mathrm{~km}$ from -13.5 to $+113.1 \mathrm{~km} \mathrm{~s}^{-1}$, as observed with the JCMT. The position of the protostar is marked by a red star. The positions targeted by GREAT/SOFIA and HIFI/Herschel are marked by blue stars. The size of the telescope HPBW (15") is indicated by the white circle. 


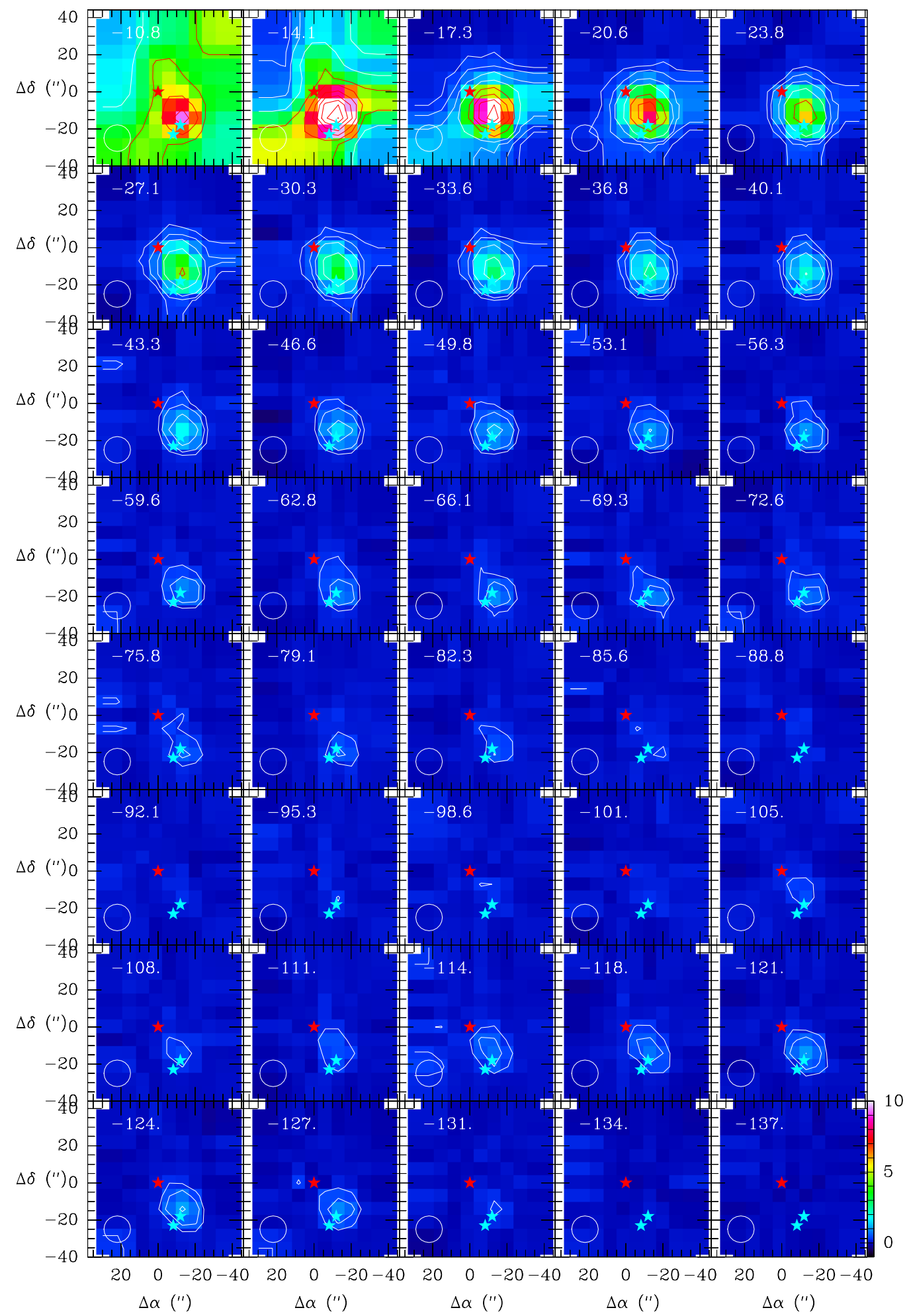

Fig. A.4. Map of the CO $J=3-2$ emission integrated in channels of $3.25 \mathrm{~km}$ from -137 to $-10.3 \mathrm{~km} \mathrm{~s}^{-1}$, as observed with the JCMT. The position of the protostar is marked by a red star. The positions targeted by GREAT/SOFIA and HIFI/Herschel are marked by blue stars. The size of the telescope HPBW $\left(15^{\prime \prime}\right)$ is indicated by the white circle. 


\section{Appendix B: Excitation conditions in the outflow cavity}

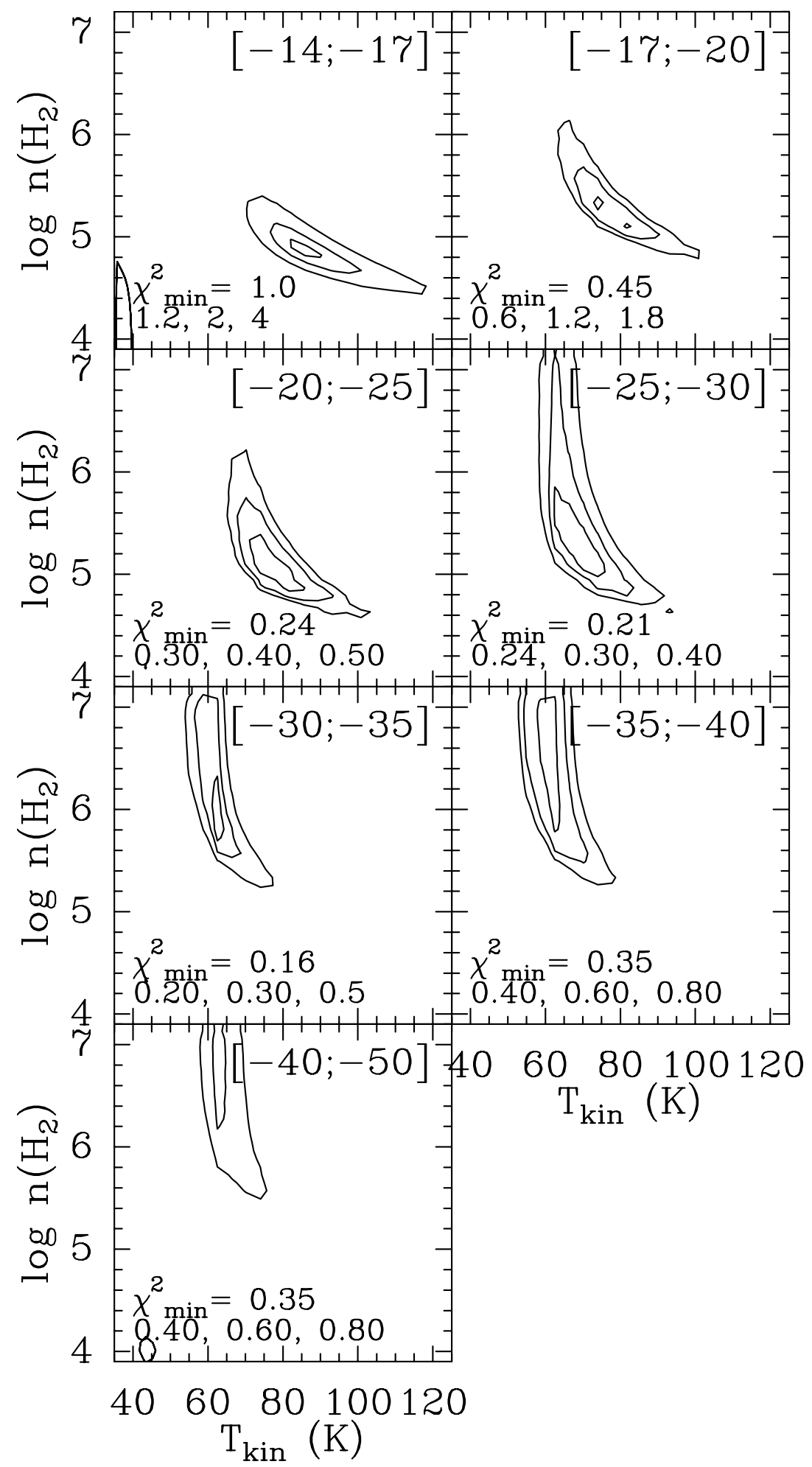

Fig. B.1. $\chi^{2}$ distribution of LVG slab models for different velocity intervals in the low-excitation gas of the outflow cavity. The considered velocity intervals are indicated in each panel. The minimum value $\chi_{\min }^{2}$ and the contour levels are indicated in each panel. 


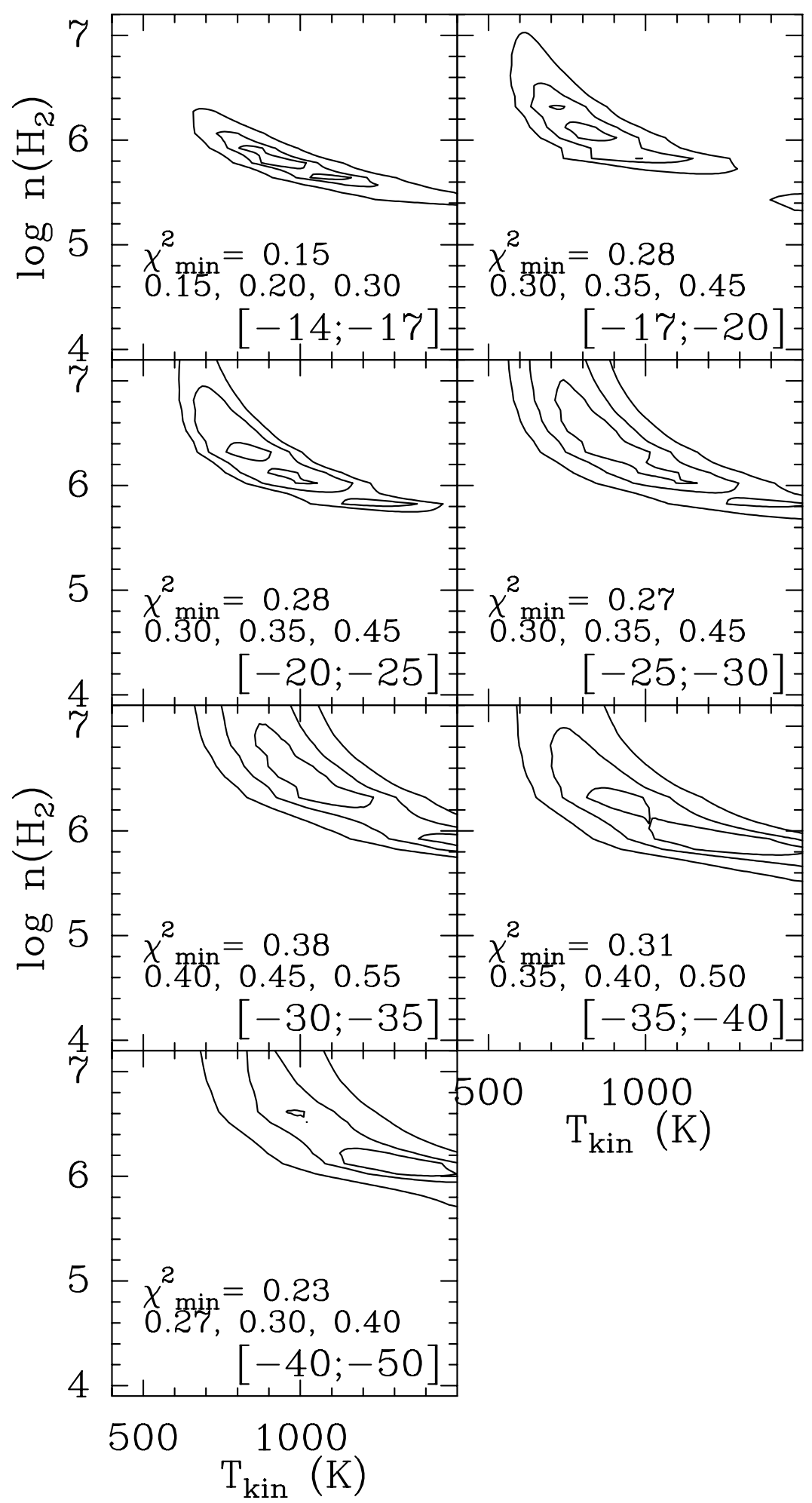

Fig. B.2. $\chi^{2}$ distribution of LVG slab models for different velocity intervals in the high-excitation gas of the outflow cavity. The considered velocity intervals are indicated in each panel. The minimum value $\chi_{\min }^{2}$ and the contour levels are indicated in each panel. 
B. Lefloch et al.: CO emission in the Cep E outflow: the outflow cavity, the bowshock, and the jet

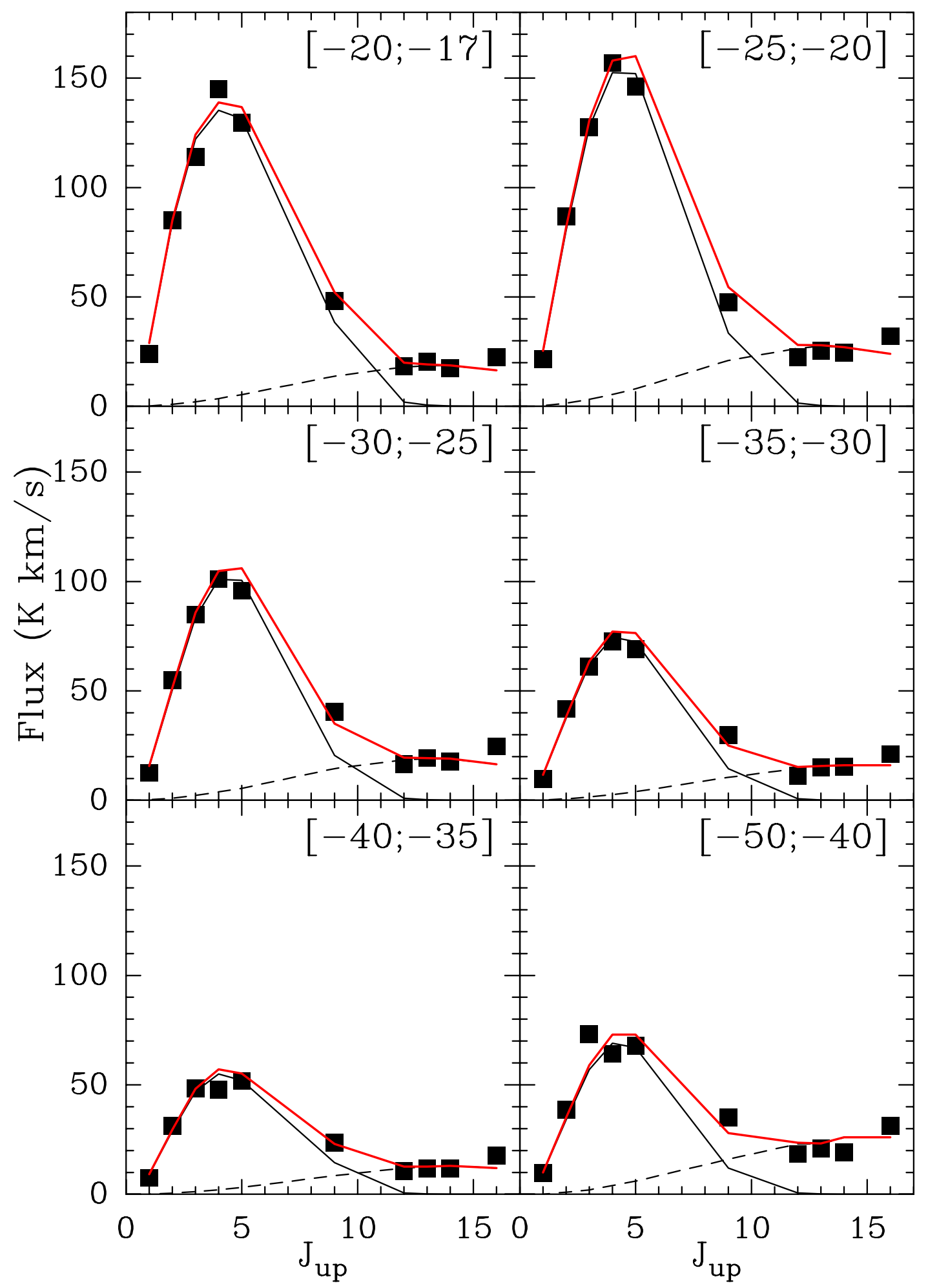

Fig. B.3. Best fitting solutions of the CO spectral line flux distribution as a function of the rotational number of the transition from our LVG analysis with the MADEX radiative transfer code. The fits to the low- and high-excitation components are shown by black solid and dashed lines, respectively. The fit to the total flux is shown in red. 